\title{
Article
}

\section{Enhancing biopharmaceutical performance of an anticancer drug by long chain PUFA based self- nanoemulsifying lipidic nanomicellar system.}

Kaur Khurana, Rajneet, Beg, Sarwar, Burrow, Andrea Julie, Vashishta, Rakesh K, Katare, O P, Kaur, Satvinder, Kesherwani, Prashant, Singh, Kamalinder and Singh, Bhupinder

Available at http://clok.uclan.ac.uk/19999/

Kaur Khurana, Rajneet, Beg, Sarwar, Burrow, Andrea Julie, Vashishta, Rakesh K, Katare, O P, Kaur, Satvinder, Kesherwani, Prashant, Singh, Kamalinder ORCID: 0000-0001-7325-0711 and Singh, Bhupinder (2017) Enhancing biopharmaceutical performance of an anticancer drug by long chain PUFA based self-nanoemulsifying lipidic nanomicellar system. European Journal of Pharmaceutics and Biopharmaceutics . ISSN 0939-6411

It is advisable to refer to the publisher's version if you intend to cite from the work. http://dx.doi.org/10.1016/j.ejpb.2017.09.001

For more information about UCLan's research in this area go to http://www.uclan.ac.uk/researchgroups/ and search for < name of research Group >.

For information about Research generally at UCLan please go to http://www.uclan.ac.uk/research/

All outputs in CLoK are protected by Intellectual Property Rights law, including Copyright law. Copyright, IPR and Moral Rights for the works on this site are retained by the individual authors and/or other copyright owners. Terms and conditions for use of this material are defined in the policies page. 


\section{Accepted Manuscript}

Enhancing biopharmaceutical performance of an anticancer drug by long chain PUFA based self-nanoemulsifying lipidic nanomicellar system

Rajneet Kaur Khurana, Sarwar Beg, Andrea Julie Burrow, Rakesh K Vashishta, OP Katare, Satvinder Kaur, Prashant Kesherwani, Kamalinder K Singh, Bhupinder Singh

PII:

DOI:

Reference:

To appear in:

Received Date:

Revised Date:

Accepted Date:
S0939-6411(17)30760-9

http://dx.doi.org/10.1016/j.ejpb.2017.09.001

EJPB 12586

European Journal of Pharmaceutics and Biopharmaceutics

21 June 2017

22 August 2017

2 September 2017

Please cite this article as: R. Kaur Khurana, S. Beg, A. Julie Burrow, R.K. Vashishta, O. Katare, S. Kaur, P. Kesherwani, K.K. Singh, B. Singh, Enhancing biopharmaceutical performance of an anticancer drug by long chain PUFA based self-nanoemulsifying lipidic nanomicellar system, European Journal of Pharmaceutics and Biopharmaceutics (2017), doi: http://dx.doi.org/10.1016/j.ejpb.2017.09.001

This is a PDF file of an unedited manuscript that has been accepted for publication. As a service to our customers we are providing this early version of the manuscript. The manuscript will undergo copyediting, typesetting, and review of the resulting proof before it is published in its final form. Please note that during the production process errors may be discovered which could affect the content, and all legal disclaimers that apply to the journal pertain. 


\title{
Enhancing biopharmaceutical performance of an anticancer drug by long chain PUFA based self-nanoemulsifying lipidic nanomicellar system
}

\author{
Rajneet Kaur Khurana, ${ }^{a}$ Sarwar Beg, ${ }^{\text {a Andrea Julie Burrow, }}{ }^{\mathrm{b}}$ Rakesh K Vashishta, ${ }^{\mathrm{c}}$

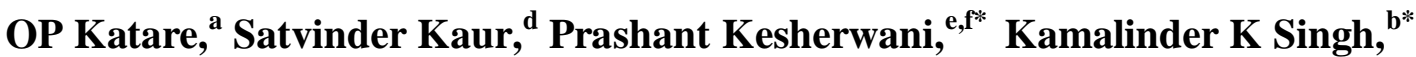 \\ Bhupinder Singh ${ }^{\mathrm{a}, g^{*}}$
}

${ }^{a}$ University Institute of Pharmaceutical Sciences, UGC Centre of Advanced Studies, Panjab University, Chandigarh 160014, India

${ }^{b}$ School of Pharmacy and Biomedical Sciences, University of Central Lancashire, Preston PRI 2HE, UK

${ }^{c}$ Department of Histopathology, Post Graduate Institute of Medical Education and Research, Chandigarh 160012, India

${ }^{d}$ GHG Khalsa of Pharmacy, Gurusar Sadhar, Ludhiana 141104, India

${ }^{e}$ Department of Pharmaceutical Technology, International Medical University, Bukit Jalil Kuala Lumpur, Malaysia

${ }^{f}$ Pharmaceutics Division, CSIR-Central Drug Research Institute, Lucknow, UP, 226031, India

${ }^{g}$ UGC-Centre of Excellence in Applications of Nanomaterials, Nanoparticles and Nanocomposites (Biomedical Sciences), Panjab University, Chandigarh 160014, India Graphical abstract:
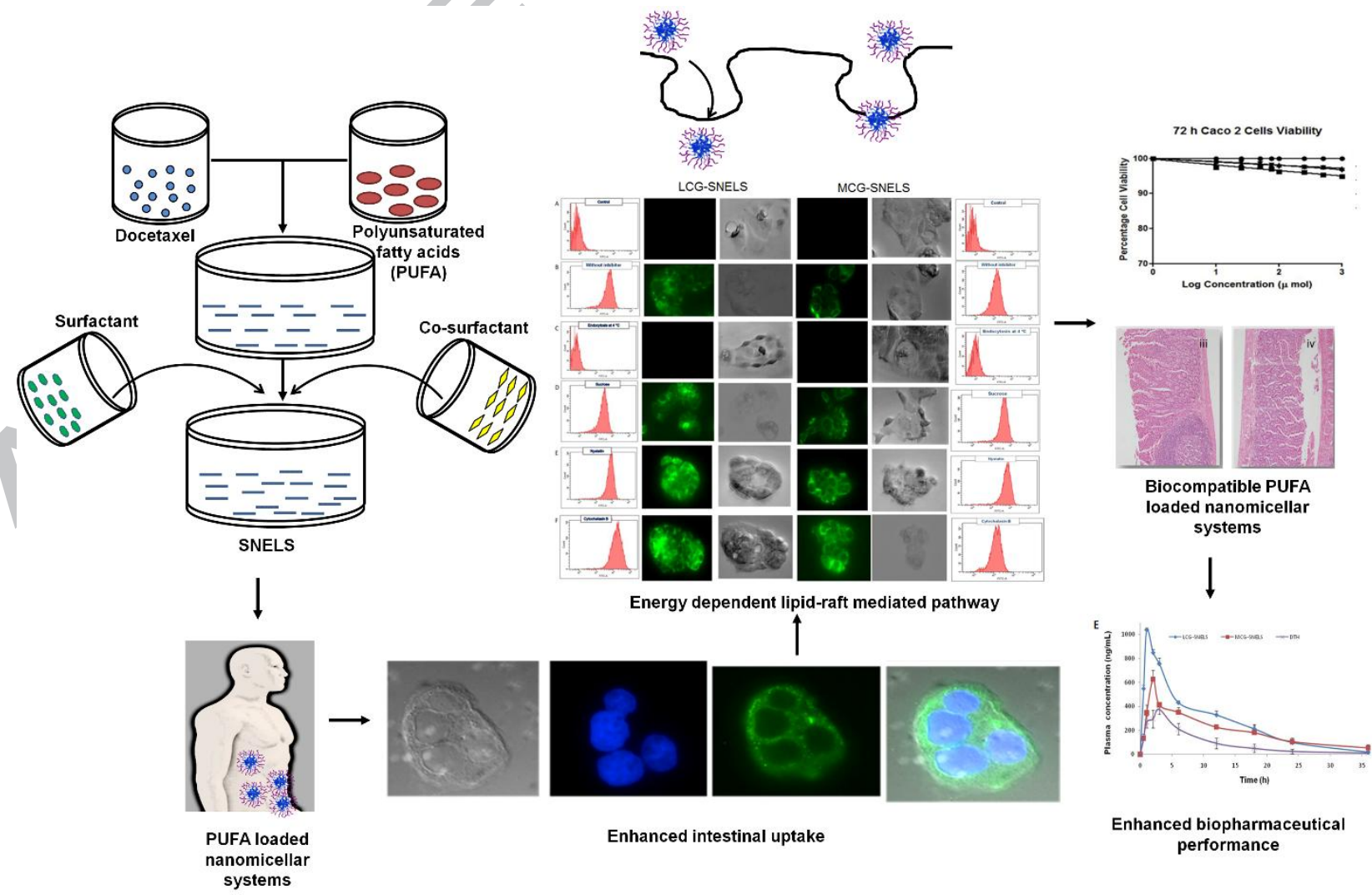
*Author for correspondence:

\section{Prof. Bhupinder Singh}

University Institute of Pharmaceutical Sciences, UGC Centre of Advanced Studies,

Panjab University, Chandigarh

160014, India

Email: bhupindersinghbhoop@ @rediffmail.com Ph: +91-172-2534103; 2541142 Fax: 2543101
Dr. Prashant Kesharwani

Department of Pharmaceutical Technology International Medical University, Kuala Lumpur, 57000 MALAYSIA Email: prashantdops@gmail.com Prashant pharmacy04@rediffmail.com Tel / Fax: +91-7999710141

Disclosures: There is no conflict of interest and disclosures associated with the manuscript.

\section{ABSTRACT}

The aim of this study was to develop polyunsaturated fatty acid (PUFA) long chain glyceride (LCG) enriched self-nanoemulsifying lipidic nanomicelles systems (SNELS) for augmenting lymphatic uptake and enhancing oral bioavailability of docetaxel and compare its biopharmaceutical performance with a medium-chain fatty acid glyceride (MCG) SNELS. Equilibrium solubility and pseudo ternary phase studies facilitated the selection of suitable LCG and MCG. The critical material attributes (CMAs) and critical process parameters (CPPs) were earmarked using Placket-Burman Design (PBD) and Fractional Factorial Design (FFD) for LCG- and MCG-SNELS respectively, and nano micelles were subsequently optimized using I- and D-optimal designs. Desirability function unearthed the optimized SNELS with $\mathrm{T}_{\mathrm{emul}}<5 \mathrm{~min}, \mathrm{D}_{\mathrm{nm}}<100 \mathrm{~nm}, \operatorname{Rel}_{15 \mathrm{~min}}>85 \%$ and Perm $_{45 \mathrm{~min}}>75 \%$. The SNELS demonstrated efficient biocompatibility and energy dependent cellular uptake, reduced P-gp efflux and increased permeability using bi-directional Caco-2 model. Optimal PUFA enriched LCG-SNELS exhibited distinctly superior permeability and absorption parameters during ex vivo permeation, in situ single pass intestinal perfusion, lymphatic uptake and in vivo pharmacokinetic studies over MCG-SNELS.

Keywords: PUFA lipids; docetaxel; Quality by Design; bi-directional permeability; P-gp efflux; Lymphatic uptake; Oral bioavailability. 


\section{Introduction}

Regardless of several innovative delivery systems and possible routes to deliver drugs, oral drug administration has been highly sought-after route owing to the ease of administration, better safety, low cost of therapy and improved patient compliance [1]. Oral delivery of anticancer drugs, in this context, is a key step towards providing "chemotherapy at home", keeping patients out of hospitals with no needles, reduced travel/clinic time and decreased staffing requirements of healthcare professionals. Of late, a time and motion study in the UK suggested that adopting oral over intravenous chemotherapy would lead to a 7-fold enhancement in the number of patients treated. Besides, oral chemotherapy is particularly attractive in palliative setting too [2].

Docetaxel trihydrate (DTH), a blockbuster semi-synthetic antineoplastic drug, is derived from the precursor, 10-deacetyl baccatin III. It belongs to family of taxanes, primarily recommended for the treatment and management of diverse kinds of cancer, especially breast, ovarian, prostate, head, neck and small cell lung carcinomas [3]. Despite its enormous utility, the deliverability of DTH has been so far commercialized in the form of injectable formulations (e.g., Taxotere ${ }^{\circledR}$, Duopafei ${ }^{\circledR}$ ) only, with no oral product of DTH yet available in the market [4]. Oral delivery of DTH is known to pose multiple drug delivery challenges including its low bioavailability. Though with $\log \mathrm{P}$ of 4.26 DTH should have culminated in moderate permeability, but high P-gp efflux, extensive hepatic first-pass effect, considerable 
gut wall metabolism by cytochrome P450 isozymes and high acid lability, coupled with poor aqueous solubility $(0.12 \mathrm{mg} / \mathrm{mL})$, all of which leads to hindrance in attaining the desired plasma levels of the drug [5].

Although diverse oral drug delivery technologies like solid dispersions [6], inclusion complexes [7], cocrystals [8], lyophilized products [9], have been reported for DTH in literature, yet none of these formulations have yielded high degree of fruition owing to their limitation to solubility enhancement characteristics only.

Polyunsaturated fatty acids (PUFA)-based drug delivery systems have been increasingly investigated for improved bioavailability owing to the enhanced oral drug absorption. It is reported that PUFA-rich lipids containing omega-3 and omega- 6 fatty acids solubilize the drugs due to micellization leading to absorption through lymphatic pathways. Despite, several investigations have been performed, yet there are barely any reports on PUFA rich formulations for enhancement of anticancer activity of various chemotherapeutic drugs including DTH till date $[10,11]$. Present study was therefore taken to prepare selfnanoemulsifying lipidic systems (SNELS) of DTH using PUFA lipids.

Lipid-based drug delivery systems, especially the self-nanoemulsifying lipidic systems (SNELS) have been widely explored to surmount the challenges and issues of low oral bioavailability in the past a few decades $[12,13]$ These SNELS tend to improve the oral absorption by producing self-nanoemulsifying micellar systems of the drug for attaining desired plasma drug levels by holistically surmounting the aforesaid hiccups. The unique properties of lipids, namely, their physicochemical diversity, biocompatibility, and ability to enhance oral bioavailability of poorly water soluble lipophilic drugs through selective lymphatic uptake have made them attractive candidates as carriers for oral delivery $[14,15]$ SNELS also exhibits stellar features like enhanced permeability, circumvention of first-pass 
effect due to facilitated drug absorption through lymphatic pathways, and inhibition of P-gp efflux, thus improving bioavailability [16].

Lately, holistic Quality by Design (QbD) paradigms has permeated into the general practice and industrial environs in order to obtain a robust and in-built quality products, as per the FDA recommendations $[17,18]$. Considered as a science and risk-based approach, QbD provides rational understanding of the critical material attributes (CMAs) and/or critical process parameters (CPPs) affecting the critical quality attributes (CQAs) of the formulation. It employs the principles of quality risk management (QRM), factor screening and Design of Experiments (DoE) for identifying the plausible risk(s) and interaction(s) associated among the process and material variables [19]. Development of robust SNELS formulations tend to involve myriad factors, including the type, nature, chain length of lipids and surfactants, which influence the biopharmaceutical performance of the drug(s) [20]. Although there are some investigations reported on self-nanoemulsifying systems of DTH demonstrating improvement in its dissolution performance and oral bioavailability [21], however, there are hardly any reports on systematic investigation determining the effect of chain length of lipidic constituents on the fate of DTH permeation, absorption and lymphatic uptake following oral administration of SNELS, and investigating the underlying mechanisms responsible for it. The current research work, therefore, endeavors for systematic QbD-based development of the SNELS for augmenting the oral bioavailability of DTH employing long-chain (LC) PUFA glycerides and comparing it with medium-chain (MC) C8/C10 fatty acid glycerides $[22,23]$. The developed lipidic formulations were evaluated through a series of in vitro experiments including bidirectional Caco-2 permeability model, cellular uptake on Caco-2 with effect of inhibitors, in situ intestinal perfusion studies and in vivo pharmacokinetic and lymphatic uptake studies for investigating the underlying mechanisms responsible for augmenting the oral bioavailability of DTH over the pure drug.

\section{Materials and methods}




\subsection{Materials}

DTH was provided ex-gratis by M/s Cipla Limited, Mumbai, India. Capryol 90, Lauroglycol FCC, Lauroglycol 90, Labrasol, Maisine 35-1, Labrafac PG, Transcutol HP, Acrysol K160 and Labrafil 2136 were received as the gift samples from M/s Gattefosse, Saint-Priest, France. Tocopherol polyethylene glycol succinate (TPGS) and Peceol was supplied ex-gratis by M/s BASF Mumbai, India. Captex 200, Captex 300, Capmul MCM and Capmul PG8 were received as the gift samples from M/s Abitec Corp., Wisconsin, USA. All other materials and chemicals procured for the studies were of analytical grade, and were used as such as obtained. Animals were procured from Panjab University's Central Animal House under the approval number PU/IAEC/S/14/104.

\subsection{Methods}

\subsubsection{Defining the QTPP and CQAs}

As per QbD-based paradigms, the objectives for development of SNELS were defined in terms of quality target product profile (QTPP) and CQAs [23]. The risk assessment studies were carried out to identify the CMAs and/or CPPs for DTH-loaded SNELS plausibly affecting the CQAs of drug product. The fishbone diagram identifies many possible causes for an effect or problem. Further, the risk assessment studies were carried out by constructing the risk estimation matrix (REM) for identifying the potential risk(s) or highly influential material attributes (MAs) and/or process parameters (PPs) on the CQAs of the SNELS [24]. The details regarding the selection of QTPP and CQAs have been elaborated in Supplementary Table 1 and Supplementary Table 2, respectively.

\subsubsection{Equilibrium solubility studies in lipids and emulgents}

Equilibrium solubility of DTH was determined in various lipids encompassing a broad spectrum of chemistry including monoglycerides/diglycerides/triglycerides mixtures, propylene glycol esters, fatty acid esters and fatty acids. LC lipidic excipients investigated for solubility studies included, Maisine ${ }^{\mathrm{TM}}$ 35-1 (a blend of partially digested long-chain 
glycerides: $33.5 \%$ monoglycerides, $50.9 \%$ diglycerides, and $14.7 \%$ triglycerides, predominantly of linoleic acid (51.1\%, omega-6 C18: 2) and oleic acid (32.8\%, omega-9 C18: 1) was employed to fabricate the DTH-SNELS), PeceolTM (glyceryl monooleate) LauroglycolTM $^{\mathrm{TM}}$ FC (propylene glycol monolaurate type I), Lauroglycol ${ }^{\mathrm{TM}} 90$ (propylene glycol monolaurate type II), ethyl oleate and oleic acid. MC lipids included Capmul MCM (a blend of partially digested medium-chain glycerides: $60.7 \%$ monoglycerides, $33.1 \%$ diglycerides, and $4.4 \%$ triglycerides, predominantly caprylic acid (82.8\%, C8), Capmul PG8, (propylene glycol monocaprylate), Captex 200 (propylene glycol dicaprylate/dicaprate), Captex 300 (glycerol caprylate caprate), and Labrafac PG (propylene glycol dicaprylocaprate). Solubility of drug was also determined in surfactants, Acrysol K-160 (polyoxyl 40-hydrogenated castor oil), Labrafil 2136 (linoleoyl polyoxyl-6 glycerides), Labrasol (PEG-8 glyceryl caprylate/caprate), vitamin E TPGS (d--tocopheryl polyethylene glycol 1000 succinate), Tween 40 (polyoxyethylenesorbitan monopalmitate) and Tween 80 (polyoxyethylene 20 sorbitan monooleate), and cosolvents, viz. Transcutol HP (diethylene glycol monoethyl ether), PEG 200 and PEG 400. Shake flask method was employed to determine the solubility [25], wherein an excess quantity of DTH was incorporated in vials containing $0.5 \mathrm{~g}$ of different lipids and emulgents, and incubated in a water bath shaker $(\mathrm{M} / \mathrm{s}$ Rivotec, Mumbai, India) for $72 \mathrm{~h}$ at $37^{\circ} \mathrm{C}$ with 25 strokes/min. The supersaturated mixtures were centrifuged at $10,000 \mathrm{rpm}(559 \times \mathrm{g})$ for $10 \mathrm{~min}$ in order to separate the supernatant fraction, which was diluted with methanol and analyzed using an already developed and validated UPLC method for estimating the solubilized fraction of DTH [26].

\subsubsection{Phase titration studies}

Water titration method was employed to construct the pseudoternary phase diagrams, with each of the two selected lipids, i.e., a LC PUFA glyceride (LCG i.e., Maisine ${ }^{\mathrm{TM}}$ 35-1) and a MC C8/C10 fatty acid glyceride (MCG i.e., Capmul MCM) with emulgent/co-solvent mixture $\left(S_{\text {mix }}\right.$ in the ratios of 1:0, 1:1, 2:1 and 3:1) in the ratio varying between 1:9 and 9:1 
$\mathrm{w} / \mathrm{w}$, to estimate the maximum nanoemulsion region. Constant visual observation and weight measurements were performed during titration of the lipid and $S_{\operatorname{mix}}$ with water for phase clarity [27]. Pseudoternary phase diagrams were constructed to delineate the nanoemulsion region using PCP-Disso software version 3.0 (University of Bradford, West Yorkshire, UK).

\subsubsection{Factor screening studies}

Placket-Burman Design (PBD) and Fractional Factorial Design (FFD) were employed to screen out "vital few" factors from "possible so many product and process variables", in order to formulate LCG- and MCG-SNELS, respectively. The selected material attributes (MAs) finally screened were amounts of lipid, emulgent and cosolvent and the process parameters (PPs) were type of mixing, emulsion stirring speed, emulsion stirring time and temperature. The low and high levels of various MAs and PPs used during factor screening studies of the LCG- and MCG-SNELS are described in Supplementary Table 3 a and 3 b, respectively. A total of twelve formulations using PBD for LCG-SNELS, and eight formulations using FFD for MCG-SNELS were prepared by mixing DTH with the mixture of selected lipids and emulgents, and were evaluated for the chosen CQAs, viz. globule size $\left(\mathrm{D}_{\mathrm{nm}}\right)$, percent drug release in $15 \min \left(\operatorname{Rel}_{15 \mathrm{~min}}\right)$ and percent dissolution efficiency $\left(\mathrm{T}_{\mathrm{emul}}\right)$. Using half-normal plots and Pareto charts, highly significant main effects were delineated either as CMAs or CPPs.

\subsubsection{QbD-based formulation development and optimization}

After identifying the CMAs actually influencing the performance of SNELS, I- and D-optimal mixture designs were employed for systematic optimization of the LCG- and MCG-SNELS with sixteen runs and five replicates in each design, using Design Expert ${ }^{\circledR}$ software version 9.0.1 (M/s Stat-Ease, Minneapolis, USA). The selected CMAs were amounts of Maisine-35-1 (LCG) and Capmul MCM (MCG) both as $\mathrm{X}_{1}$, amount of Tween 80 
$\left(\mathrm{X}_{2}\right)$ and amount of Transcutol HP $\left(\mathrm{X}_{3}\right)$. The ratios of three components were adjusted to make the weight of upto $1 \mathrm{~g}$ of the formulation. DTH $(20 \mathrm{mg})$ was dissolved in molten mixture of lipid, surfactant and cosolvent at $40^{\circ} \mathrm{C}$, followed by vortex mixing for $5 \mathrm{~min}$. All the prepared formulations were evaluated for $\mathrm{D}_{\mathrm{nm}}, \mathrm{T}_{\mathrm{emul}}, \mathrm{Rel}_{15 \min }$ and percent permeated in 45 $\min \left(\right.$ Perm $\left._{45 \mathrm{~min}}\right)$ as the CQAs. Further, optimization data analysis was carried out by multiple linear regression analysis (MLRA) by modelling to fit in second-order quadratic polynomial model along with added interaction terms to correlate the CMAs with CQAs. The suitability of model fitness was evaluated using P-value, coefficient of correlation $(\mathrm{R})$ and predicted error sum of squares (PRESS). The generated 3D-response surface plots enabled understanding of the relationship and/or interactions among the studied CMAs and their influence on the CQAs.

\subsection{Characterization of SNELS formulations}

\subsubsection{Globule size}

Aliquots of $1 \mathrm{~mL}$ of both types of SNELS formulations were diluted in $250 \mathrm{~mL}$ of 0.1 $\mathrm{N} \mathrm{HCl}(\mathrm{pH}$ 1.2) and were subjected to globule size and zeta potential measurement employing Zetasizer ZS 90, M/s Malvern Instruments, Worcestershire, UK.

\subsubsection{Emulsification time}

Aliquots of $1 \mathrm{~mL}$ of each of SNELS formulations were studied for self-emulsification time by pouring the liquid formulation drop wise to $250 \mathrm{~mL}$ of $0.1 \mathrm{~N} \mathrm{HCl}(\mathrm{pH} 1.2)$, while stirring at $50 \mathrm{rpm}$ and maintained at $37 \pm 0.5^{\circ} \mathrm{C}$, using a USP XXXI Apparatus II (DS 8000, M/s Lab India Instruments, Mumbai, India).

\subsubsection{Transmission electron microscopy (TEM)}

Optimized SNELS formulations were diluted in a manner analogous to that mentioned in Section 2.3.2, and subsequently stained with $1 \%$ phosphotungstic acid for 1 min, before subjecting for TEM analysis (JEM-2100 F, M/s Jeol, Tokyo, Japan).

\subsubsection{In vitro drug release}


Dialysis bag (12 KDa) method was employed to carry out in vitro drug release studies employing $250 \mathrm{~mL}$ of $0.1 \mathrm{~N} \mathrm{HCl}$ containing $1 \%$ sodium lauryl sulphate for $1 \mathrm{~h}$, stirred continuously at $50 \mathrm{rpm}$ and $37 \pm 5^{\circ} \mathrm{C}$ [28]. Aliquots of $5 \mathrm{~mL}$ were withdrawn at definite time points and replenished with fresh dissolution medium to maintain sink conditions, and analyzed by previously validated and reported method using Ultra Performance Liquid Chromatography (UPLC) by us [26].

\subsubsection{Ex vivo permeation studies}

Ex vivo permeation studies were carried out by method already been reported by us previously [27]. Briefly, the entire small intestine of sacrificed rats was excised and washed with Kreb's Ringer Buffer (KRB). Jejunum section was cut and ligated with thread to one end of a glass rod in order to evert it. The gut sac was filled with KRB solution and placed thereafter in a bath containing $50 \mathrm{~mL}$ of same buffer maintained at $37 \pm 0.5^{\circ} \mathrm{C}$. LCG- and MCG-SNELS (each containing drug equivalent to $20 \mathrm{mg}$ of DTH) were poured in buffer outside the gut sac. Aliquots of $1 \mathrm{~mL}$ each were withdrawn at definite time intervals, followed by replacement with fresh KRB solution and analyzed using UPLC to determine the amount of drug permeated and Perm $45 \mathrm{~min}$.

\subsubsection{In vitro lipolysis studies}

In vitro lipolysis studies were performed to measure and compare the release of the DTH into the aqueous phase from LCG- and MCG-SNELS. As the model was designed to mimic the dynamic physiological conditions, previously reported by Dahan and Hoffman [29], where upon agitation, SNELS will form emulsion and disperse in the digestion medium. The model was built around a temperature-controlled $\left(37^{\circ} \mathrm{C}\right)$ vessel containing $100 \mathrm{~mL}$ of digestion buffer, composition of which was adapted from the previous report [29]. The digestion buffer contained calcium chloride, sodium chloride, sodium hydroxide, tris-maleate and most importantly pancreatin. 
One gram each of LCG- and MCG-SNELS formulations (each containing $20 \mathrm{mg}$ of DTH) was dispersed in $100 \mathrm{~mL}$ of prepared pre-warmed digestion buffer in order to activate the lipase enzyme. A pH meter was used to maintain the $\mathrm{pH}$ at 6.8 with $1 \mathrm{M} \mathrm{NaOH}$ throughout the experiment [30], i.e., for $60 \mathrm{~min}$. Constant $\mathrm{pH}$ monitoring was required as triglycerides were hydrolyzed during lipolysis, to release free fatty acids, thereby decreasing the $\mathrm{pH}$ of the medium. Aliquots of $5 \mathrm{ml}$ were withdrawn at periodic intervals of $0,5,10,15$, 30 and 60 min and the sampled volume was replaced by blank digestion buffer. In order to inhibit lipolysis, the withdrawn samples were inhibited with $20 \mu \mathrm{L}$ of 1 M 4bromophenylboronic acid (4-BPBA), a lipase inhibitor [31] and centrifuged at 75,000 rpm $\left(6.28 \times 10^{5} \mathrm{~g}\right)$ for $10 \mathrm{~min}$ at $4{ }^{\circ} \mathrm{C}$ in a Beckmann-Coulter Optima ${ }^{\mathrm{TM}}$ MAX Ultracentrifuge (Fullerton, CA). Drug content was analyzed at each time point using UPLC [26].

\subsection{Caco-2 cell line studies}

\subsubsection{Caco-2 cell culture}

Caco-2 cell line (cell passages between 40 and 55) was procured from European Collection of Authenticated Cell Cultures (ECACC), Public Health England, Salisbury, England. Cells were grown in EMEM containing $2 \mathrm{mM}$ glutamine, $1 \%$ (v/v) non-essential amino acid solution (NEAA), supplemented with $10 \%(\mathrm{v} / \mathrm{v})$ heat-inactivated foetal bovine serum (FBS), at $37{ }^{\circ} \mathrm{C}$ in an atmosphere of $5 \% \mathrm{CO}_{2}(\mathrm{ESCO}, \mathrm{UK})$ in the tissue culture flasks (75 $\mathrm{cm}^{2}$; Thermo, USA). Once-cultured cells were $90 \%$ confluent, these were trypsinized employing $0.1 \%$ trypsin-EDTA solution.

\subsubsection{Cell viability assay}

For cell viability studies, $1 \times 10^{3}$ cells $/ \mathrm{cm}^{2}$ were seeded on 96 -well plate (Thermo Fischer Scientific, Roskilde, Denmark) for overnight. Before conducting the experiment, the medium was aspirated from the cells, and $90 \mu \mathrm{L}$ of both LCG- and MCG-SNELS, diluted with medium were added to each identified well. After 24,48 and $72 \mathrm{~h}$ of treatment, $10 \mu \mathrm{L}$ of PrestoBlue $^{\mathrm{TM}}$, a cell viability reagent (Invitrogen, MD, USA) was poured in each well, and 
the cells were incubated for $1 \mathrm{~h}$. The fluorescence was determined using a microtiter plate reader at the excitation/emission wavelength of 535/612 nm, respectively.

\subsubsection{Bidirectional permeability assay}

Caco-2 monolayers were grown for 19-21 days on inserts. Briefly, $2 \times 10^{4}$ cells $/ \mathrm{cm}^{2}$ were seeded on sterile polyester membrane and polystyrene plates $(12 \mathrm{~mm}$ diameter inserts, $0.4 \mu \mathrm{m}$ pore size) (M/s Corning Inc., Costar ${ }^{\circledR}, \mathrm{NY}$, USA). On every alternative day, transepithelial electrical resistance (TEER; Millicell@-ERS-2 Voltohm meter, M/s Millipore Corporation, Billerica, MA, USA) value was measured to monitor the growth of Caco-2 monolayers and the extent up to, which the cellular tight junctions were formed [32]. On 19th to 21 st day, as the cell monolayers reached the constant TEER value, i.e., starting from $150 \mathrm{ohm}$ to about $1700 \mathrm{ohm}$, these were used for experimentation. The permeation of both LCG- and MCG-SNELS was studied from the apical to basolateral direction $(\mathrm{A} \rightarrow \mathrm{B})$ and basolateral to apical $(\mathrm{B} \rightarrow \mathrm{A})$ direction in Caco-2 cells. Caco-2 cell monolayers were equilibrated in pre-warmed $\mathrm{HBSS}$ at $37^{\circ} \mathrm{C}$ for $30 \mathrm{~min}$ just before commencing the experiment. On the apical side, $1.0 \mathrm{~mL}$ of pre-warmed HBSS containing plain drug, and LCG- and MCG- SNELS loaded with DTH was added, each containing drug in the dose of $100 \mu \mathrm{mol}$. Subsequently, $0.5 \mathrm{~mL}$ of blank HBSS was added to the basolateral side. Similarly, the same formulations were also added on basal side and blank HBSS was added to apical side to better understand the permeability assay. After incubation for $0.5,1,2$ and $4 \mathrm{~h}, 0.2 \mathrm{~mL}$ of medium at the basolateral side was withdrawn and replaced with fresh medium to investigate $\mathrm{A} \rightarrow \mathrm{B}$ transport. Similarly, $0.2 \mathrm{~mL}$ of medium at the apical side was withdrawn and replaced with fresh medium for investigation of $\mathrm{B} \rightarrow \mathrm{A}$ transport. Drug concentrations from both the sides were determined by HPLC analysis and the amount of drug permeated, thus calculated, and was plotted as a function of time. Apparent permeability coefficient (Papp; $\mathrm{cm} / \mathrm{s})$ was computed from the slope of the linear plot, using the following equation: 
$\operatorname{Papp}=\frac{\Delta Q / \Delta t}{\operatorname{Co} \times A}$

where, $\Delta Q / \Delta t$ is the steady state flux indicating linear appearance rate of mass in the basolateral side; $\mathrm{C}_{0}$ is initial concentration of drug at the apical (for $\mathrm{A} \rightarrow \mathrm{B}$ transport) or basolateral (for $\mathrm{B} \rightarrow \mathrm{A}$ transport); and $A$ is the surface area of the membrane $\left(\mathrm{cm}^{2}\right)$.

\subsubsection{Intracellular uptake and visualization}

The qualitative uptake studies were carried out by seeding $1 \times 10^{5}$ cells/well on cover slips in 6-well tissue culture well plates (Thermo Scientific Nunc Multidish 6, Roskilde, Denmark), and were allowed to adhere for overnight [32]. The cells were incubated for $0.5,1$, 2 and $4 \mathrm{~h}$ with $0.078 \mu \mathrm{M}$ Rh-123-loaded LCG- and MCG-SNELS, diluted with media. Following the specified incubation period, the respective cover slips on which the Caco-2 cells adhered were removed. After washing with PBS, the cells were fixed with paraformaldehyde and washed thrice with PBS prior to mounting on Vectashield ${ }^{\circledR}$ mounting medium with DAPI. Cells were observed on a modified Zeiss Cell Observer imaging system using Zeiss EC Plan-Neofluar 40x/1.3 Oil DIC objective. Rh-123 and DAPI were imaged using a GFP/DAPI filter set with excitation/emission wavelength of 450-490nm/500-550nm and 335-383nm/420-470nm, respectively. Analysis of the images was carried out using Zeiss ZEN desk imaging software for analysing the cellular uptake as a function of fluorescence intensity.

\subsubsection{Quantitative cellular uptake by flow cytometry}

Cellular uptake studies were carried for evaluating the influence of time-dependent uptake of the developed formulations on Caco- 2 cells. For time-dependent uptake, $1 \times 10^{5}$ cells/well were seeded and incubated in 6-well culture plate for overnight. At predetermined time intervals, i.e., 0.5, 1, 2, 4 h, cells treated with Rh-123-loaded LCG- and MCG-SNELS diluted with medium were removed and washed twice with PBS (pH 7.4). Further, the cells 
were trypsinized $(0.1 \%)$ and the cell extracts were centrifuged at $10,000 \mathrm{rpm}(559 \times \mathrm{g})$ for 10 min and the supernatant was discarded. The cells were re-suspended in cold PBS and subjected to flow cytometry (BD FACSAria, USA) for estimating the fluorescence intensity employing BD filter of FITC 530/30 and PE Texas Red 610/20 employing BD FACSDiva software.

\subsubsection{Uptake mechanistic evaluation studies}

To analyze the mechanism of uptake of SNELS, Caco- 2 cells $\left(1 \times 10^{5} /\right.$ well $)$ were treated with various endocytosis inhibitors for $30 \mathrm{~min}$ prior to the experiment for selectively blocking the endocytosis pathways. To study the clathrin-dependent endocytosis, sucrose (0.45 M) was employed, while for caveolae-mediated endocytosis, cells were pretreated in medium supplemented with nystatin $(5 \mu \mathrm{g} / \mathrm{mL})$. Cytochalasin B $(5 \mu \mathrm{g} / \mathrm{mL})$ was used for assessing the macropinocytosis or phagocytosis, uptake followed by inhibition of energydependent endocytosis by storing the cells-loaded with formulation at $4^{0} \mathrm{C}$ [33]. The cells were further treated and incubated with Rh-123 loaded SNELS for $1 \mathrm{~h}$. Subsequent to experimentation; the media containing SNELS was removed and washed with PBS. Further, cells were made to survive in PBS, till these were viewed under live microscope for quantitative assessment of the intensity difference with all the inhibitors.

\subsubsection{P-gp efflux assay}

Overexpression of P-gp on Caco-2 cells is well documented in literature [34]. P-gp confers resistance by preventing enough accumulation of anticancer drugs within the cell, thereby avoiding their cytotoxic or apoptotic effects [35]. For evaluating the P-gp efflux, the multi-drug resistance dye efflux assay kit (M/s Chemicon International, USA) was employed. The efflux activity was measured to determine the intracellular accumulation of the fluorescent dyes, Rh-123 and $\mathrm{DiOC}_{2}$, to access the inhibitory activity of SNELS on MDR1 and BCRP transporters, respectively. Caco-2 pellet containing $2.5 \times 10^{5}$ cells was incubated 
with Rh-123 alone and Rh-123 with vinblastine, Rh-123 with LCG- and MCG-SNELS respectively for $1 \mathrm{~h}$ at $37{ }^{\circ} \mathrm{C}$. All the formulations were suspended in RPMI-1640 medium (M/s Sigma-Aldrich, UK). Likewise, to estimate the potential of the developed SNELS formulation for blocking the activity of $\mathrm{BCRP}$ transporters, $\mathrm{DiOC}_{2}$ dye was employed. Similarly, Caco-2 cells $\left(2.5 \times 10^{5}\right)$ were incubated with $\mathrm{DiOC}_{2}$ alone, $\mathrm{DiOC}_{2}$ and vinblastine, DiOC $_{2}$ with LCG- and MCG-SNELS respectively at $37^{\circ} \mathrm{C}$. The cells were washed with prewarmed PBS and the fluorescence intensity was measured in a TECAN fluorescence microplate reader at an excitation wavelength of $485 \mathrm{~nm}$ and an emission wavelength of 530 $\mathrm{nm}$.

\subsection{Lymphatic uptake studies}

The lymphatic route enables the orally administered lipidic delivery systems to reach systemic circulation by intestinal lymphatic transport, thus evading hepatic first-pass metabolism through portal vein. Therefore, in vivo lymphatic uptake studies were carried out to investigate the extent of oral absorption of DTH from LCG- vis-à-vis MCG-SNELS [36]. Briefly, overnight fasted unisex Wistar rats (200-250g each) were divided into three groups, each group containing three animals. Various treatment formulations viz. pure drug suspension, LCG- and MCG-SNELS, each containing $20 \mathrm{mg} / \mathrm{ml}$ of oral dose of DTH, were administered to the animals through peroral route equivalent to the body weight of rats at dose of $20 \mathrm{mg} / \mathrm{kg}$. At predetermined time intervals of $1,3,6$ and $12 \mathrm{~h}$, the rats were anaesthetized by intraperitoneal injection of thiopental sodium $(50 \mathrm{mg} / \mathrm{kg}$ dose $)$, followed by cannulation of the mesenteric lymph duct with the help of a polyethylene tube $(15 \mathrm{~cm}$ length, i.d. $0.58 \mathrm{~mm}$, o.d. $0.96 \mathrm{~mm}$ ) [37]. The lymph (approx. $0.2 \mathrm{~mL}$ ) was periodically collected in eppendorf tubes at the specified time intervals and the drug was extracted by adding suitable volume of acetonitrile, followed by centrifugation at 10,000 $\mathrm{rpm}(2,236 \times \mathrm{g})$ for $10 \mathrm{~min}$. The supernatant organic fraction was collected and the organic phase was evaporated. The remaining dry residue was subsequently reconstituted in mobile phase and analyzed through a 
previously developed and validated UPLC method of DTH in lymph. The concentration of drug uptaken in lymph was estimated from the calibration plot of the drug in lymph and the obtained data were plotted versus time.

\subsection{In situ single pass intestinal perfusion (SPIP) and histopathology studies}

In situ SPIP were performed as per the procedure reported in literature [20]. Unisex Wistar rats (250-280 g), previously fasted for overnight, were used for the study. Animals were randomly assigned to the different experimental groups, i.e., DTH solution, DTH with verapamil, LCG- and MCG-SNELS respectively. Each group consisted of three animals and the experiments were carried out employing an in-house fabricated assembly. Anesthesia was induced by an intraperitoneal injection of thiopental sodium in the dose of $50 \mathrm{mg} / \mathrm{kg}$ of the body weight of the rats. The abdomen was incised up to $2-4 \mathrm{~cm}$ to create cannulated inlet and outlet with polyethylene tubing at proximal part on the jejunum. It was further perfused with lukewarm KRB solution. Subsequently, the intestine was perfused with LCG- and MCGSNELS, both at a rate of $0.2 \mathrm{~mL} \cdot \mathrm{min}^{-1}$. After $30 \mathrm{~min}$, an aliquot of $1 \mathrm{~mL}$ each was periodically withdrawn, every 15 minutes. The perfusate was extracted with diethyl ether and subsequent centrifugation at $10,000 \mathrm{rpm}(559 \times \mathrm{g})$ for $10 \mathrm{~min}$. Following extraction, DTH concentration was determined using UPLC analysis at $230 \mathrm{~nm}$ [26]. The permeability parameters like, effective permeability $\left(\mathrm{P}_{\text {eff }}\right)$, wall permeability $\left(\mathrm{P}_{\text {wall }}\right)$, and absorption parameters like fraction absorbed $(\mathrm{Fa})$ and absorption number $(\mathrm{An})$ were calculated. In order to investigate the statistical significance difference among various parameters, one-way ANOVA was applied using GraphPad Prism ver 5.0 (M/s GraphPad Inc., California, USA).

Following perfusion studies, histopathological evaluation of the intestinal segments was conducted. On the excised small intestine segment, for all the groups, by fixing a part in $10 \% \mathrm{w} / \mathrm{v}$ neutral buffered formalin solution for atleast $24 \mathrm{~h}$. The process of fixation helps the tissue to prevent decomposition, putrefaction, and autolysis and withstand subsequent processing. Further, the tissue samples were processed by embedding these in paraffin wax, 
which were subsequently sectioned into slices of $5 \mu \mathrm{m}$ thickness and stained with hematoxylin and eosin (H\&E) for microscopic examination (Olympus, Tokyo, Japan).

\subsection{In vivo pharmacokinetic studies}

In vivo single-dose pharmacokinetic studies were performed in parallel design. The animals were divided into three groups ( $\mathrm{n}=6$ each), i.e., Group I administered with pure DTH solution, while the animals in Group II and III were administered with LCG- and MCGSNELS, each at oral dose of $20 \mathrm{mg} / \mathrm{kg}$ equivalent to body weight of rats, respectively [38]. Formulations were administered to each rat by oral gavages and subsequently, blood (100 $\mu \mathrm{L}$ ) was withdrawn from each animal's retro-orbital plexus at specified time intervals of 0.5 , $1,2,3,6,12,18,24$ and $36 \mathrm{~h}$. Further, plasma was extracted by centrifugation at 10,000 rpm $(559 \times \mathrm{g})$ for $10 \mathrm{~min}$, and analysed for DTH using UPLC analysis [26].

Pharmacokinetic parameters were computed using WinNonlin software version 5.0 (M/s ThermoScientific, USA) [20] and their statistical validity was ratified by two-way ANOVA using GraphPad Prism software version 5.0 (M/s GraphPad Inc., California, USA).

\section{Results and discussion}

\subsection{Risk assessment studies}

Supplementary Table 4 displays the risk estimation matrix (REM) constructed to identify the risk(s) associated with each CMAs and/or CPPs. The FMEA suggested that the amount of lipid, emulgent and co-emulgent were associated with high risk, while type of mixing process, stirring speed, stirring time and temperature were associated with medium risk. It depicted that type of lipids and emulgents were also associated with low risk. Based on the REM, the high to medium risk variables were subjected to factor screening studies for identifying the potential CMAs actually affecting the CQAs of SNELS formulations.

\subsection{Preliminary screening of excipients}


Equilibrium solubility studies revealed that mixed mono-, di-, triglycerides of both LCG (Maisine ${ }^{\mathrm{TM}}$, 35-1: $40.71 \mathrm{mg} \cdot \mathrm{mL}^{-1}$; (Supplementary Figure 1 A) and MCG (Capmul MCM: $37.93 \mathrm{mg} \cdot \mathrm{mL}^{-1}$ : (Supplementary Figure $1 \mathrm{~B}$ ) showed higher solvation capacity for DTH than for other lipids. This is in agreement with an earlier report, where mono-, di-, triglycerides have shown higher solubility for a range of drugs than the corresponding triglycerides [39]. Between LCG and MCG, LCG showed higher solubility probably due to longer lipid chain length [40] responsible for maximal solubilization of DTH. Minimum solubility for DTH was exhibited by Lauroglycol FCC (1.51 mg.mL $\mathrm{mL}^{-1}$ ) (Supplementary Figure $1 \mathrm{~A}$ ) and Captex ${ }^{\circledR} 300\left(1.45 \mathrm{mg} \cdot \mathrm{mL}^{-1}\right)$, respectively (Supplementary Figure $\left.1 \mathrm{~B}\right)$.

The nonionic emulgent, Tween 80 (Supplementary Figure $1 \mathrm{C}$ ), was found to be most suitable for attaining maximal drug solubilization $\left(85.21 \mathrm{mg} \cdot \mathrm{mL}^{-1}\right)$ with high emulsification capacity and with added advantage of low toxicity during oral intake [20]. Amongst cosolvents, maximum solubility was exhibited by Transcutol HP (95.55 mg. $\mathrm{mL}^{-1}$ ), while minimum by PEG $200\left(6.26 \mathrm{mg} \cdot \mathrm{mL}^{-1}\right)$ (Supplementary Figure $\left.1 \mathrm{C}\right)$. Excipients demonstrating highest solubility were selected to achieve high loading capacity of DTH in the SNELS formulation. It is already demonstrated that high solubility of drug in the SNELS is not merely required for maintaining the drug in a solubilized form in the oily phase of the resultant dispersion but also for preventing the precipitation of the drug in the gastrointestinal (GI) lumen after dilution with GI fluids [41].

\subsection{Phase titration studies}

Phase titration studies were performed with both Maisine-35-1 and Capmul MCM as LCG and MCG lipids, respectively, with Tween 80 and Transcutol HP as the corresponding surfactant-cosurfactant mixture $\left(\mathrm{S}_{\text {mix }} ; 1: 0,1: 1,2: 1\right.$ and $\left.3: 1\right)$, in the varying ratios ranging between 1:9 and 9:1 for delineating the nanoemulsion region. The constructed pseudoternary phase diagrams delineate a good nanoemulsion region at the said $S_{\text {mix }}$ ratios of LCG and MCG, respectively. The wide nanoemulsion region can be ascribed to the efficient 
emulsification of both the lipids in the presence of surfactant and cosolvent [20]. The inference drawn from the studies revealed that on increasing the amount of Tween 80 in $S_{\text {mix }}$ ratio from 1:1 to 2:1 ratios, a wider nanoemulsion region was observed (Figure 1). Further increase in the Tween 80 concentration narrowed down the nanoemulsion region, as is clearly evident from the phase diagrams for both the lipids employed for SNELS formulations. Also, the studies revealed that on increasing the amount of Transcutol HP in the $S_{\text {mix }}$, increase in the nanoemulsion region was observed ostensibly due to its enhanced solubilization capacity and surface-wetting property. Further, it is evident from the ternary diagrams that the MCG formed a wider microemulsion region as compared to LCG as is illustrated in Figure 1. High polarity and low hydrophobicity of the MCG primarily seem to account for such a marked difference [42]. As MCG exhibits higher mobility characteristics on the lipid/water interfaces, it is documented to possess the capacity to get rapidly hydrolyzed by up taking large amount of water, thus forming wider nanoemulsion region [12]. On the basis of maximal nanoemulsion region observed during the above studies for the LCG and MCG, $S_{\text {mix }}$ ratio of 2:1 was found to be highly suitable.

\subsection{Factor screening studies}

High and medium risk factors were screened for LCG-SNELS and MCG-SNELS employing PBD and FFD, respectively. Linear polynomial equations generated employing mathematical modeling revealed no significant interaction(s) amongst the MAs and PPs. The plots and charts indicated the influence of each MA/PP on the studied CQAs (i.e., $\mathrm{D}_{\mathrm{nm}}$, $\% \mathrm{DE}_{5 \min }, \mathrm{T}_{\mathrm{emul}}, \mathrm{Rel}_{15 \mathrm{~min}}$, and MDT). Therefore, it was concluded that the values lying above the t-value limit and Bonferroni line are highly influential, i.e., concentration of Capmul MCM, Maisine-35-1, Tween 80 and Transcutol HP [Figure 2 (i) and (ii)]. Both of the lipids, surfactant and co-solvent showed notable effect on $\mathrm{Re}_{15 \min }, \mathrm{MDT}, \mathrm{D}_{\mathrm{nm}}$ and $\mathrm{T}_{\mathrm{emul}}$, while Tween 80 showed higher influence on the values of $\% \mathrm{DE}_{5 \min }$. Hence, being highly influential, these factors were chosen as CMAs, and subsequently employed for systematic optimization 
of both LCG-SNELS and MCG-SNELS. Further, as rest of the factors caused only negligible influence on the CQAs, these were fixed at constant value for subsequent optimization studies.

\subsection{QbD-based model development and response surface analysis (MLRA)}

A second-order quadratic equation was generated using MLRA method, and ten coefficients were obtained for each of the CQAs (Eq. 2). High values of R, ranging between 0.965 and 0.998 , coupled with lower values of PRESS revealed excellent goodness of fit, $Y=\beta_{1} X_{1}+\beta_{2} X_{2}+\beta_{3} X_{3}+\beta_{4} X_{1} X_{2}+\beta_{5} X_{1} X_{3}+\beta_{6} X_{2} X_{3}+\beta_{7} X_{1} X_{2} X_{3}+\beta_{8} X_{1} X_{2}\left(X_{1}-X_{2}\right)+\beta_{9} X_{1} X_{3}\left(X_{1}-X_{3}\right)+\beta_{10} X_{2} X_{3}\left(X_{2}-X_{3}\right)$ ...Eq.(2)

where, $\mathrm{Y}$ is the response variable; $\beta_{1}$ to $\beta_{4}$ are the coefficients of linear model terms; $\beta_{5}$ to $\beta_{7}$ are the coefficients of quadratic model terms, $\beta_{8}$ to $\beta_{10}$ are the coefficients of cubic model terms with added interaction terms; while $X_{1}, X_{2}, X_{3}$ represent the factors employed during the current optimization studies. The mathematical equations generated for each CQAs revealed synergism and antagonism amongst selected CMAs, depending upon the sign of the polynomial coefficients.

The constructed response surface plots facilitated comprehensive understanding regarding influence of CMAs on the studied CQAs. The 3D-response surface plots (Figure 3 A and 3 B) illustrate the higher influence of surfactant and moderate effect of co-solvent on $\mathrm{D}_{\mathrm{nm}}$. With increase in the levels of surfactant and cosolvent, a nonlinear trend was noticed on $\mathrm{D}_{\mathrm{nm}}$. In contrast, a linear increase was observed with increasing levels of Maisine-35-1 and Capmul MCM on $\mathrm{D}_{\mathrm{nm}}$. The smallest globule size was obtained at lower levels of lipids and cosolvent, and higher levels of surfactant, respectively.

Figure $3 \mathrm{C}$ and $3 \mathrm{D}$ reveal higher influence of lipids and emulgent on $\mathrm{T}_{\mathrm{emul}}$, while the influence of co-emulgent was found to be insignificant. Short emulsification time was observed at low levels of lipids and intermediate levels of surfactants. This can be primarily 
ascribed to the spontaneous emulsification of Maisine-35-1 and Capmul MCM in water by Tween 80, leading eventually to faster emulsification and lower emulsification time.

Figure $3 \mathrm{E}$ and $3 \mathrm{~F}$ portray $3 \mathrm{D}$-response surface plot for $\mathrm{Rel}_{15 \mathrm{~min}}$ exhibiting a quick descending trend on increasing the amount of lipids, while the trend was found to be curvilinear with Tween 80. Moreover, increasing the concentration of Transcutol HP showed increase in the values of $\operatorname{Rel}_{15 \mathrm{~min}}$. Overall, both the SNELS formulations showed nearly complete drug release within $30 \mathrm{~min}$ at intermediate levels of lipids and Tween 80 and high levels of Transcutol HP, respectively.

Figure $3 \mathrm{G}$ and $3 \mathrm{H}$ depicts a response surface plot for Perm $45 \min$. A curvilinear trend was observed for Perm $45 \min$ on increasing the levels of the lipids, emulgents and co-emulgent. However, the effect LCG was quite conspicuous indicating increase in the permeability with increase in the Maisine35-1 (Figure 3 G). The highest Perm $45 \min _{\text {Was }}$ observed at relatively higher levels of Maisine-35-1 and Capmul MCM and intermediate levels of Tween 80 and Transcutol HP.

By and large, prevalence of distinct nonlinear trends in these response surfaces unequivocally indicate involvement of interactions among the studies excipients, thus corroborating the remarkable utility and superiority of systematic QbD-enabled paradigms over the traditional one variable at one time (OVAT) approach.

\subsection{Characterization of the prepared LCG and MCG SNELS}

\subsubsection{Globule size measurement}

The mean globule size for all the SNELS prepared as per the experimental design, were found to range between 15 and $110 \mathrm{~nm}$, affirming the nanostructured character of the developed SNELS containing either LC or MC lipids. However, the mixed micelles of LCGSNELS were observed to be significantly larger $(101 \mathrm{~nm}$; $\mathrm{P}<0.001)$ Supplementary data Figure 2 A) than MCG-SNELS (16 nm; Supplementary data Figure 2 B). The LC lipids have 
already been reported to result in polydisperse emulsions with significantly lower size [43] ascribed to their longer fatty chain length than that of MC lipids. Further, the smaller globules were found to be formed at lower concentrations of lipid, higher concentrations of surfactant and intermediate concentrations of cosolvent present in the formulations. Varying the proportions of surfactant and co-surfactant, attributed the condensation and stabilization of interfacial film, resulting in the formation of smaller droplets, in consonance with previous findings [44]. However, on adding more of co-surfactant the film generally expands and increases the globule size [45].

\subsubsection{Self-emulsification time}

$\mathrm{T}_{\mathrm{emul}}$ values of all the formulations ranged between 135 and $205 \mathrm{sec}$ (i.e., $<5 \mathrm{~min}$ ) for both LCG- and MCG-SNELS. Lower values of $\mathrm{T}_{\mathrm{emul}}$ indicated spontaneity of emulsification producing nanoemulsions with transparent bluish tinge. In general, formulation containing higher concentration of Tween 80 exhibited faster emulsification leading to absolute miscibility of lipids in the aqueous phase by micellar solubilisation [41].

\subsubsection{In vitro drug release studies}

Figures 4 (A) and 4 (B) portray the in vitro release profiles of DTH from LCG SNELS and MCG SNELS, respectively using dialysis bag method. Almost $80 \%$ of drug release was observed within first 30 minutes, and nearly $90 \%$ drug released within $1 \mathrm{~h}$. Since in vitro dissolution profiles observed for both the types of LCG- and MCG-based nanoemulsified formulations were almost analogous, the lipid chain length was not found to affect the drug release from the SNELS appreciably [27]. However, it was interesting to note that the formulations with higher levels of surfactants released the drug faster and better [27].

\subsubsection{Zeta potential studies}

The values of zeta potential for LCG- and MCG-SNELS were found to be $-29.8 \mathrm{mV}$ and $-35.0 \mathrm{mV}$, respectively (Supplementary Figure 3). Indicative of the degree of repulsion 
between adjacent and similarly charged particles, zeta potential tends to delineate the stability potential of the formulation [27]. Nanosized systems characterized by high values of zeta potential have been reported to be highly stable by virtue of their resistance against aggregation and/or coalescence [20].

\subsubsection{Ex vivo permeation studies}

The ex vivo permeation of optimized formulation in 45 minutes was found to be more than approximately $85 \%$ for LCG SNELS and $70 \%$ for MCG SNELS, which can certainly be attributed to the enhanced absorption of LCG SNELS owing to its longer chain length [20].

\subsection{Search for the optimum formulation and validation of QbD}

Various CQAs were "traded off" to attain the desired objectives, i.e., smaller $D_{n m}$, minimum $T_{\text {emul }}$, with maximum release in $15 \min \left(\operatorname{Rel}_{15 \mathrm{~min}}\right)$ and higher permeability in 45 $\min \left(\right.$ Perm $\left._{45 \mathrm{~min}}\right)$. In order to attain the stated objectives, the selection criteria embarked upon to search the optimized formulation were $\mathrm{T}_{\text {emul }}<5$ minutes, $\mathrm{D}_{\mathrm{nm}}<100 \mathrm{~nm}$ and $\operatorname{Rel}_{15 \mathrm{~min}}>80 \%$. Numerical optimization methodology was carried out for identifying the optimum formulation, where all the CQAs exhibited desirability close to unity. Further, Figure $4 \mathrm{C}$ and $4 \mathrm{D}$ portray the optimum formulations of LCG- and MCG-SNELS demarcated in the design space overlay plot. The optimum LCG-SNELS contained Maisine-35-1 (338 mg), Tween 80 (434 mg) and Transcutol HP (227 mg), with the values of CQAs as $\mathrm{D}_{\mathrm{nm}}$ of $98 \mathrm{~nm}, \mathrm{~T}_{\mathrm{emul}}$ of 1.3 min, $\operatorname{Rel}_{15 \min }$ of $75 \%$ and Perm $_{45 \min } 82 \%$. On the other hand, the optimum MCG-SNELS contained Capmul MCM (353 mg), Tween 80 (440 mg) and Transcutol HP (205 mg), with the values of CAQs as $\mathrm{D}_{\mathrm{nm}}$ of $106 \mathrm{~nm}, \mathrm{~T}_{\mathrm{emul}}$ of $1.2 \mathrm{~min}, \mathrm{Rel}_{15 \mathrm{~min}}$ of $84 \%$ and $\mathrm{Perm}_{45 \mathrm{~min}}$ of $60 \%$. Also, TEM imaging (Figure $4 \mathrm{E}$ ) of optimized LCG-SNELS revealed the size range between 60.4 and $70.7 \mathrm{~nm}$, while MCG-SNELS exhibited the size range of 11.7 to $21.0 \mathrm{~nm}$ [Figure $4(\mathrm{~F})]$. 


\subsection{In vitro lipolysis studies}

In vitro digestion profile of DTH-loaded LCG- and MCG-SNELS was investigated in the prepared bio-relevant dissolution media followed by UPLC analysis for the fraction of solubilised DTH in the aqueous phase. The rate of digestion of SNELS by pancreatic lipase was estimated by measuring the consumption of sodium hydroxide $(\mathrm{NaOH})$ needed to neutralize the released fatty acids during lipolysis. The rate and extent of digestion and the phase behavior of the lipolytic products is markedly dependent on the fatty acid chain length. Similar to previous report by Sek and associates [46], post 30 minute lipid digestion on ultracentrifugation, the MCG-SNELS (Figure $5 \mathrm{C}$-i) showed complete digestion, separating into two distinct phases, i.e., aqueous and pellet fraction in a bile-salt-independent manner. The LCG-SNELS (Figure 5 C-ii), on the other hand, got separated into three phases, i.e., oily (i.e., containing undigested triglyceride and diglyceride), aqueous (i.e., containing bile salt, fatty acid and monoglyceride), and a pellet phase (i.e., containing fatty acid, presumably as an insoluble soap). However, digestion for both the formulations was observed to be quite fast at early time points. Maisine-35-1 and Capmul MCM, both being the lipase substrates [47], exhibited similar lipolysis rates as indicated by insignificant difference $(\mathrm{P}>0.01)$ in $\mathrm{NaOH}$ consumption of LCG- and MCG-SNELS (Figure 5A) [48]. The release of DTH from both these SNELS formulations (Figure 5 B) showed a similar release pattern without any significant difference $(\mathrm{P}>0.05)$

\subsection{Caco-2 cell line assays}

\subsubsection{Cell viability assay}

Quite often, there could be impairment of Caco-2 monolayers, plausible ascribable to cytotoxicity of lipids and/or surfactants, eventually leading to false reporting of augmentation in drug permeation. Therefore, it is essential for any promising lipidic formulations to have good tolerance at the site(s) of absorption, and quite vital to test the optimum formulations for 
cytotoxicity prior to further biological investigation [49]. Figure 6 (A, B and C) depicts the $\log$ concentration versus percent viability profile for the Caco-2 cells incubated with free DTH, LCG- and MCG-SNELS, respectively. PrestoBlue was used as the cell viability indicator as it is considered to yield consistent, reliable and quicker results than other reagents [50]. The percent cell viability for Caco-2 cells with PrestoBlue was found to be $>96 \%$ for formulations of both the types of lipid and at all the studied concentrations, i.e., 10 , $25,50,75,100,250,500$ and $1000 \mu \mathrm{mol}$, without any significant change $(\mathrm{p}>0.05)$ between these. The studies ratified the safe and biocompatible nature of the developed SNELS.

\subsubsection{Bidirectional permeability assay}

In vitro bidirectional Caco-2 model has been extensively explored to investigate the intestinal absorption and cytotoxicity of the drugs and their formulations, as these cells differentiate into functionalized epithelial barrier [51]. Further, it was indeed important to access the permeability of the formed nanomicelles across the intestinal epithelial barrier. DTH being quite prone to P-gp efflux [52], the strategy adopted in the current studies was not only to study the $\mathrm{A} \rightarrow \mathrm{B}$ permeation but also to investigate the $\mathrm{B} \rightarrow \mathrm{A}$ transport. The bidirectional permeability assay was carried out for determining the concentration of DTH in the apical and basolateral compartments following incubation of the Caco-2 cells with different treatments. As DTH is a substrate of P-gp, it tends to efflux out from the cells, thus leading to drug resistance. Figure $6 \mathrm{D}$ illustrates the apparent permeability $\left(\mathrm{P}_{\text {app }}\right)$ of DTH during the bi-directional permeability studies performed for different treatment formulations at varied time-points. Since the cells were seeded, the TEER value was constantly monitored to predict the growth of the monolayer of Caco-2, while measurement during the experimentation was performed to investigate any possible damage of the formed monolayer [53]. The recorded TEER values for growing monolayer increased from 150 to $1600 \Omega \bullet \mathrm{cm}^{2}$ in around 21 days. Further, on incubation with all the treatments, significant reduction 
$(<10 \%$; $\mathrm{P}<0.05)$ in TEER values was observed vis-à-vis the control cells, indicating that the formulations affect tight junctions [54]. This can be ascribed to the presence of polysorbate, which has already been documented to affect the tight junctions of the Caco-2 monolayers and thus account for paracellular transport [55].

The results revealed that nanomicelles formed from the DTH-loaded SNELS showed a six-fold increase in permeation $\left(\mathrm{P}_{\text {app }}, 12.9 \times 10^{-6} \mathrm{~cm} / \mathrm{sec}\right)$ as compared to plain DTH solution $\left(\mathrm{P}_{\mathrm{app}}, 1.9 \times 10^{-6} \mathrm{~cm} / \mathrm{sec}\right)$ within $4 \mathrm{~h}$ of the studied time period (Figure 6 D). This construed enhancement in permeability of DTH-loaded SNELS, is ascribable to lipidic and emulsifying excipients employed for the preparation of SNELS. Such excipients tend to improve the permeability of drug ostensibly owing to their lipophilic nature along with ability to form nanomicelles after emulsification in aqueous phase [56]. Moreover, higher permeability of SNELS across the Caco-2 cells can be related to the P-gp efflux pump inhibition, governing the transport and permeability of DTH from the SNELS [57]. Interestingly, it was observed that $\mathrm{P}_{\mathrm{app}}$ increased with time, which could be attributed to the ability of Tween 80 [58] and Transcutol HP [21] to block the P-gp efflux across the intestinal barrier, resulting consequently in increased permeation with time [32].

The results also clearly demonstrated the superiority of LCG-SNELS to MCG-SNELS owing to the longer chain length of the lipids employed in the former, which facilitate faster internalization of the drug molecules across the membrane of Caco- 2 cells. Additionally PUFAs are known to cause reduction in pump activity of MDR1/P-gp which would have contributed to increased permeability of LC PUFA based SNELS [59]. Effux ratio of DTH, a known P-gp substrate [60], was reduced to 0.20 and 0.25 , when administered as LCG- and MCG-SNELS, respectively compared to 0.84 for the drug alone. This could be assigned to inhibition of P-gp triggered efflux by Tween 80 [61] and Transcutol HP [62], and the increased permeability owing to nature of lipidic excipients and their characteristic chain length. 
Overall, the Caco-2 cells studies revealed that SNELS can augment the permeation of DTH owing to P-gp efflux inhibition and facilitate faster permeation across the Caco- 2 cells for enhanced drug bioavailability.

\subsubsection{Qualitative and quantitative cellular uptake}

Figure 7 depicts the fluorescent images of Caco-2 cells incubated with various formulations labelled with fluorescent dye Rh-123. Figures $7 \mathrm{~B}$ and $7 \mathrm{C}$ reveal the presence of fluorescence inside the cells treated with Rh-123-loaded MCG- and LCG- SNELS at $1 \mathrm{~h}$, which later increased remarkably at $4 \mathrm{~h}$ (Figures $7 \mathrm{D}$ and $7 \mathrm{E}$ ), thus substantiating the timedependent and efficient internalization of sub-micron emulsion droplets by Caco-2 cells. Also, the qualitative estimation of the fluorescence intensity divulges superior uptake potential of LCG-SNELS (Figure $7 \mathrm{C}$ and $7 \mathrm{E}$ ) to MCG-SNELS (Figure $7 \mathrm{~B}$ and $7 \mathrm{D}$ ) corresponding to the same time-points. The quantitative flow cytometry results showed timedependent increase in fluorescence for both the LCG- and MCG-SNELS (Figure 7 F), indicating maximum cellular uptake at $4 \mathrm{~h}$. The results observed from the uptake studies were found to be in consonance with the permeability studies, thus corroborating the superiority of LCG-SNELS over the MCG-SNELS for potentiating drug absorption of DTH.

\subsubsection{Mechanism of cellular uptake}

The endocytosis of a nanoparticle is an active and energy-dependent process, which can be inhibited by low temperature. Therefore the difference in cellular uptake at $37{ }^{\circ} \mathrm{C}$ and $4{ }^{\circ} \mathrm{C}$ at $1 \mathrm{~h}$ was qualitatively and quantitatively compared via fluorescence microscopy and flow cytometry assay. The relative intracellular fluorescence percentages of both the SNELS at $37{ }^{\circ} \mathrm{C}$ were significantly greater than that at $4{ }^{\circ} \mathrm{C}$ at the given time point, i.e., $1 \mathrm{~h}$ (Figure $8 \mathrm{~B}$ and $8 \mathrm{C}$ ). More precisely, the cellular uptake at $37{ }^{\circ} \mathrm{C}$ was about 12 - and 10 -folds higher than that at $4{ }^{\circ} \mathrm{C}$ for LCG- and MCG-SNELS at $1 \mathrm{~h}$, respectively (Figure $8 \mathrm{G}$ ). It has been reported that the active transportation on cell membranes significantly reduces at $4{ }^{\circ} \mathrm{C}$ [63]. 
Therefore, the studies demonstrate that the endocytosis of SNELS by Caco- 2 cells to be energy-dependent, involving an active process [64].

To elucidate the cellular trafficking pathway of SNELS different inhibitors blocking specific pathway were used. Hypertonic sucrose induces abnormal clathrin polymerization into empty microcages rendering clathrin unavailable for assembly into normal coated pits and inhibits endocytosis. Nystatin, a known inhibitor of caveolae-mediated endocytosis profoundly distorts the structure and functions of the cholesterol-rich membrane domain, including aberrations in the caveolar shape while cytochalasin A causes depolymerisation of the actin filaments thus disrupting the actin-mediated macropinocytosis. An endeavor has been made to qualitatively and quantitatively understand the mechanistic pathways through measurement of intensity of the fluorescence of internalized Rh-123 loaded SNELS after treatment with these pharmacological inhibitors. Figure 8 A illustrates the image for the control cells, while Figure $8 \mathrm{~B}$ reveals the intensity of fluorescence after $1 \mathrm{~h}$ incubation of Caco- 2 cells with the LCG- and MCG-SNELS formulations without any inhibitor. On the contrary, Figure 10 D to F reflects the fluorescence intensity within cells when treated with the three types of inhibitors, viz. sucrose, nystatin and Cytochalasin B. The results demonstrate that none of inhibitors had any effect on endocytosis of SNELS. The SNELS internalization was not mediated through any of these pathways. The flow cytometry (FCM) analysis endorsed these results with insignificant impact $(\mathrm{P}>0.05)$ on cellular uptake of SNELS, indicated by nearly constant fluorescence intensity of cells following treatment with the endocytosis inhibitors (Figure $8 \mathrm{G}$ ), in concurrence with a previously reported study [65]. These findings are also supported by another report by Song and associates [66], which showed pegylated polyester nanoparticles to undergo an energy-dependent, lipid raft-mediated, but caveolae-independent endocytosis in Caco-2 cells. Cellular uptake of LCG- and MCG-SNELS was found to be an energy-dependent process, but internalization occurred in a clathrin- and caveolaeindependent manner. 


\subsubsection{P-gp efflux assay}

The P-gp efflux assay revealed that both the dyes (i.e., Rh-123 and $\mathrm{DiOC}_{2}$ ), were effluxed out at $37{ }^{\circ} \mathrm{C}$, as evident from lack of intracellular accumulation of the fluorescent dye (Figure 9 A). On the other hand, incubation with P-gp efflux inhibitor, i.e., vinblastine showed that dyes may have coupled with vinblastine, thus blocking MDR1 and BCRP transporters, both at $37{ }^{\circ} \mathrm{C}$, eventually leading to higher fluorescence intensity without any efflux. Both LCG- and MCG-SNELS interfered with the microenvironment of P-gp and weakened the P-gp mediated efflux, as apparent from higher accumulation of Rh-123 and $\mathrm{DiOC}_{2}$ dyes, suggesting MDR1 and BCRP transporters were not functional. Accordingly, the excipients of both SNELS play a major role in inhibiting the P-gp efflux and significantly reduced transporter efficiencies for the P-gp substrate, i.e., $\mathrm{Rh}-123$ and $\mathrm{DiOC}_{2}$ dyes. Previous reports have shown intact internalization of self-nanoemulsifying system into Caco2 cells bypassing P-gp recognition [67].

\subsection{Lymphatic uptake studies}

Figure 9 B illustrates the lymphatic uptake of DTH after oral administration of various test formulations with respect to time. Highly statistically significant difference $(\mathrm{p}<$ 0.001) in the lymphatic uptake of LCG-SNELS was observed from the MCG-SNELS and pure DTH at all experiment time points, when administered orally in rats. LCG-SNELS showed maximal uptake (i.e., up to 3.79-fold) from the lymph in $3 \mathrm{~h}$, while the MCG-SNELS showed nearly 2.26-fold improvement in lymphatic uptake vis-à-vis pure drug suspension. Significant improvement in the uptake of drug in lymph from LCG-SNELS can be ascribed to the faster absorption and transportation of the drug preferentially through intestinal lymphatic pathways [13]. The lipids, whether LCGs or MCGs, not only facilitate the solubilization of drugs exhibiting low solubility and lipophilicity, but also increase the fraction of drug transported via intestinal lymphatic system, thereby increasing absorption from the GI tract, depending upon the chain length of lipids [68]. The superiority of the LCG- 
over MCG-SNELS could be attributed to the transit of LCGs as long-chain fatty acids and monoglycerides across the intestinal cell, where these are re-esterified to triglycerides and further incorporated into chylomicrons to be secreted from the intestinal cell by exocytosis into the lymph vessels [69]. Whereas, MCG is transported to portal blood before going to systemic circulation, where the later encounter first-pass metabolism, thus accounting for the reduced concentration of DTH in lymph [70]. However, the reasonable response of MCG is also ascribable to the formation of medium-chain saturated fatty acids, which are resynthesized or re-esterified to triacylglycerols or neutral lipids, packaged within apoprotein surface layers and again transported via the lymphatic system [71]. Hence, it can be concluded that a multitude of factors tend to influence the lymphatic absorption of drugs, including lipid chain length, partition coefficient, and above all, the formation of chylomicrons produced after digestion of lipid-based systems to facilitate higher fraction of drug to be transported through the intestinal lymphatic system, and subsequent drainage into the systemic circulation [72].

These findings are consistent with previously published work, where MCGs are reported to be more likely to be taken up into the portal system, whereas the LCGs, are directly taken up into the lymphatic system as triglycerides [33]. The long chain fatty acid also facilitate the absorption of the drug through lymphatic pathways by circumnavigating the hepatic first-pass effect, thus helping in enhancing the distribution of the drug to the lymphatic system with consequent improvement in oral bioavailability of lipophilic drugs [13].

\subsection{In situ single pass intestinal perfusion studies (SPIP)}

The in situ SPIP studies are well known model to investigate the absorption and permeation behavior of a drug, when administered using self-nanoemulsifying systems. With LCG- and MCG-SNELS, much higher magnitudes of absorptivity and permeability parameters were observed vis-à-vis pure DTH. As is evident from Figure 9 C, LCG- and 
MCG-SNELS showed significant improvement $(\mathrm{P}<0.001)$ in the absorption number $(\mathrm{An})$ by 4.5- and 3.6-fold as compared to the pure drug, respectively [14]. The value of An indicates the amount of drug transferred across the GI tract, where both the SNELS were found to be highly superior to free drug indicating improved drug absorption characteristics. Further, marked increase $(\mathrm{P}<0.01)$ in the magnitude of fraction of drug absorbed $(\mathrm{Fa})$ was observed for LCG-SNELS (i.e., 4.1-fold) and MCG-SNELS (i.e., 3.0-folds) vis-à-vis pure drug, indicating notable augmentation in the absorption potential of the drug through SNELS, ostensibly owing to the potentiation of drug absorption through lymphatic pathways by circumnavigating the hepatic first-pass effect [73].

Similarly, in case of the permeability parameters, the effective permeability ( $\mathrm{P}_{\text {eff }}$ ) also showed nearly 4.0- and 3.2-fold improvement by the LCG- and MCG-SNELS (P < 0.001 each) vis-à-vis the pure drug. Further, the SNELS formulations also showed considerably increased $(\mathrm{P}<0.01)$ values of wall permeability $\left(\mathrm{P}_{\text {wall }}\right)$, i.e., 3.1-fold by LCGSNELS, while 2.4-fold by MCG-SNELS, vis-à-vis the pure drug. The presence of surfactant, Tween 80 in the nano micellar formulations may have induced membrane perturbation and Pgp inhibition that enhanced the drug permeability [74]. Tween 80 having both lipophilic and hydrophilic properties, partitions between lipid and protein domains in the intestinal membrane disrupting its integrity and plausibly increasing the permeability of DTH [75]. Further Tween 80 is known to modulate the P-gp efflux by inhibition of protein kinase C activity and thereby reducing the phosphorylation of P-gp [76].

P-gp efflux pump expressed all along the gastrointestinal tract limits permeability of many drugs and affects their peroral absorption and bioavailability. Verapamil being a potent P-gp inhibitor was able to improve the permeation and absorption parameters of DTH but up to a limited extent [77]. The values of An and Fa were found to be significantly improved by 3.1and 2.9-folds, for LCG-SNELS and 2.5- and 2.1-folds for MCG-SNELS vis-à-vis DTH in presence of verapamil ( $\mathrm{P}<0.01$ each). Similarly, the magnitudes of Peff and Pwall increased 
by 2.2- and 1.7-folds, and 2.2- and 1.5-folds for LCG- and MCG-SNELS, respectively, with verapamil vis-à-vis pure drug (Figure $9 \mathrm{C}$ ). The superior values of Peff and $\mathrm{Pw}$ construed increase in the permeability and uptake characteristics of the drug using SNELS, plausibly owing to the enhancement in permeability of drug and inhibition of efflux by P-gp and/or BCRP transporter owing to the presence of lipidic and emulsifying excipients in the formulations, in agreement with literature [78]. Besides, the results also showed higher magnitude for enhancement in the permeability parameters by SNELS containing LCGs, plausibly owing to being omega-6 PUFA glyceride along with its higher chain length and lipophilicity as compared to the MCGs. By and large, the LCG-SNELS showed around 1.3and 1.3-folds and 1.4 and 1.3-folds augmentation in Peff, Pwall, Fa and An, respectively (P $<0.05$ each) as compared to MCG-SNELS. Previous reports have shown enhanced intestinal permeability [79] and improved oral bioavailability with LC (C18) self-emulsifying systems [80]. LCGs form larger mixed micelles than MCGs on digestion, which tend to have a higher drug solubilizing capacity leading to higher permeability and bioavailability [12]. Caliph and associates [81] have also demonstrated the effect of chain length on the permeability across the lymph。

Further, the histopathological analysis was carried out for the intestine after perfusion experiment from each group of rats. Figure 9 D (i-iv) depicts the microscopic images of the rat's small intestinal segments after exposure to (i) Control (ii) Plain DTH (iii) LCG-SNELS (iv) MCG-SNELS. Gut segments treated with the aforementioned formulations revealed normal intestinal morphology, i.e., intact brush border and villi, without any significant pathological change(s). Also, the lymphoid follicles and submucosa were found to be normal, thus ruling out any potential toxic effects. In line with Caco-2 PrestoBlue cell viability assay, the histopathological evaluation accordingly revealed safe and biocompatible nature of both, LCG- and MCG-SNELS.

\subsection{In vivo pharmacokinetic studies}


Figure 9 E depicts the plasma concentration versus time profiles of DTH, LCGSNELS and MCG-SNELS in each group ( $\mathrm{n}=6$ ). On applying one-way ANOVA, significant statistical difference $(\mathrm{P}<0.01)$ was observed among the individual plasma concentrations of both the SNELS in contrast to pure drug. The linear decline in the post- $\mathrm{T}_{\max } \log$ concentration time data further corroborated the suitability of two-compartment model kinetics. The results observed from the current studies are in accordance with the literature report [27].

Analysis of the in vivo pharmacokinetic studies in rats as per chosen twocompartment open body model (2-CBM), exhibited much higher oral bioavailability in terms of extent and rate of absorption, as depicted from various pharmacokinetic metrics like AUC, $\mathrm{C}_{\max }, \mathrm{T}_{\max }$ and $\mathrm{K}_{\mathrm{a}}$, by LCG- and MCG-SNELS vis-à-vis free drug suspension (Table 1).

Nearly 11- and 4.6-folds improvement in $\mathrm{AUC}$ and $\mathrm{C}_{\max }$, and 2.3-fold reduction in $\mathrm{T}_{\max }$ was observed for the LCG-SNELS vis-â-vis DTH suspension, respectively. Similarly, for MCG-SNELS, 8.7- and 1.8-folds augmentation in AUC and $\mathrm{C}_{\max }$, coupled with 1.2-fold reduction in $\mathrm{T}_{\max }$ values, ratified distinct improvement in the rate and extent of oral bioavailability ( $\mathrm{p}<0.001$ ), when compared with pure DTH suspension. The LCG SNELS and MCG SNELS also showed improvement (1.9- and 1.2-folds) in the values of $K_{a}$ vis-à-vis free drug suspension, respectively.

On the whole, the pharmacokinetic studies revealed superiority of formed LCG- over MCG-SNELS in increasing the oral absorption of DTH. There are several studies reported in literature indicating that drugs from formulations containing LCGs and MCGs are transported differently in the body $[20,39,56]$. Porter and Charman have reported that MCG is directly transported by the portal blood to the systemic circulation, whereas LCG is transported in the intestinal lymphatics. Therefore, lipid-based drug delivery systems containing LCGs are likely to enhance the lymphatic transport of a lipophilic drug substance like DTH (log P 4.26) because the lymphatic transport circumnavigates the hepatic first-pass metabolism of a drug. The LCG based formulations have been previously reported to furnish higher oral 
bioavailability vis-à-vis their MCG-couterparts [81]. In a nutshell, the oral bioavailability of DTH using LC PUFA glycerides SNELS will be enhanced due to combined effect of improved solubility, inhibition of P-gp efflux along with increased intestinal lymphatic transport of drug with consequent reduction of first pass metabolism. Figure 10 portrays the mechanism of uptake of LCG- and MCG-SNELS through intestinal uptake.

\section{Conclusions:}

The SNELS formulations prepared in the present studies demonstrated significant improvement in the biopharmaceutical attributes of DTH, a BCS class drug exhibiting poor aqueous solubility, low permeability and oral bioavailability. Application of QbD-based systematic development of LCG- and MCG-SNELS resulted in comprehensive understanding of the formulation and its process parameters. Among the various LCGs and MCGs investigated for preparing SNELS, the LCG constituting PUFA showed remarkable improvement in enhancement of its dissolution with inhibition of the P-gp mediated efflux and by passing first pass effect, resulting consequently in improved oral bioavailability as vividly examplified in the in vivo pharmacokinetic studies. Also, its increased permeability and absorption parameters during ex vivo permeation and in situ perfusion studies have been illucidated. The superiority of the LCG- over MCG-SNELS was also well demonstrated in lymphatic uptake studies attributed to re-esterification of LCGs to triglycerides and their incorporation into chylomicrons and secreted from the intestinal cells into the lymph vessels. In vitro lipolysis studies indicated significant difference in the release of free fatty acids and digestion between the LCG and MCG SNELS formulations. The MCG SNELS, having the shorter lipidic chain length than LCG SNELS was more completely digested. Cytotoxicity and uptake studies on Caco-2 cells revealed safe and biocompatible nature of the nanomicellar formulations along with efficient energy dependent cellular uptake which was 
independent of clathrin- and caveolin- mediated endocytic pathways. Overall, the studies corroborated enormous utility of SNELS especially prepared using LC PUFA glycerides in augmenting the oral bioavailability of DTH. The outcome of the current studies and mechanistic unravelled can successfully be extrapolated to other BCS Class II and IV drugs. Future studies will involve understanding the potential of these formulations in cancer therapeutics by investigating their cellular ineteraction in mamalian cancer cells and efficacy in suitable animal tumor model.

\section{Acknowledgments}

The author Ms Rajneet Kaur Khurana is grateful to UGC for providing financial grants to her to carry out the present work as a Research Fellow under RFMS scheme (F. No. 5-94/2007(BSR) dated 28/02/2013). The use of biomedical facilities of University of Central Lancashire is deeply acknowledged.

\section{Declaration of Interest}

Authors declare no conflict(s) interest.

\section{References}

[1] R.K. Khurana, M. Mahajan, Teenu, S. Kapoor, S. Jain, B. Singh, The sojourn from parenteral to oral taxanes using nanocarrier systems: A patent review, Recent Pat Drug Deliv Formul., 10 (2016) 44-58.

[2] R. James, C. Blanco, F. C., Saving in staff time as a result of swithing from de gramont to oral capecitabine for patients with advanced colorectal cancer., Curr Drug Metab., S83: Abstr 271. (2003). [3] M. Mahajan, R.K. Khurana, N.S. Sahajpal, P. Utreja, R. Sankar, B. Singh, S.K. Jain, Emerging strategies and challenges for controlled delivery of taxanes: A comprehensive review, Curr Drug Metab., 16 (2015) 453-473.

[4] in: US Pharmacopoeia, National Formulary. USP 37 NF32. United States. Pharmacipoeial convention Twin Broock Parkway, Rockville, 2014, pp. 2681-2685.

[5] S. Kapse-Mistry, T. Govender, R. Srivastava, M. Yergeri, Nanodrug delivery in reversing multidrug resistance in cancer cells, Front Pharmacol, 5 (2014) 159.

[6] J. Chen, L. Qiu, M. Hu, Y. Jin, J. Han, Preparation, characterization and in vitro evaluation of solid dispersions containing docetaxel, Drug Dev Ind Pharm., 34 (2008) 588-594.

[7] L. Suna, D.S. Zhoub, P. Zhangb, Q.H. Lib, P. Liu, Gemcitabine and $\gamma$-cyclodextrin/docetaxel inclusion complex-loaded liposome for highly effective combinational therapy of osteosarcoma, Int $\mathrm{J}$ Pharm, 478 (2015) 308-317.

[8] L.K. Tatini, K. Krishna Reddy, N. Someswara Rao, Vapor-induced phase transformations in docetaxel, AAPS PharmSciTech, 13 (2012) 548-555.

[9] V. Kannan, V. Kash, Docetaxel lyophilized parentaral formulation for safe drug delivery system and process of making thereof in, 2010. 
[10] P.S. Sandhu, R. Kumar, S. Beg, S. Jain, V. Kushwah, O.P. Katare, B. Singh, Natural lipids enriched self-nano-emulsifying systems for effective co-delivery of tamoxifen and naringenin: Systematic approach for improved breast cancer therapeutics, Nanomedicine., 13 (2017) 1703-1713. doi: 1710.1016/j.nano.2017.1703.1003.

[11] P.S. Sandhu, S. Beg, F. Mehta, B. Singh, P. Trivedi, Novel dietary lipid-based selfnanoemulsifying drug delivery systems of paclitaxel with p-gp inhibitor: implications on cytotoxicity and biopharmaceutical performance, Expert Opin Drug Deliv, 12 (2015) 1809-1822.

[12] C.J. Porter, N.L. Trevaskis, W.N. Charman, Lipids and lipid-based formulations: optimizing the oral delivery of lipophilic drugs, Nat Rev Drug Discov., 6 (2007) 231-248.

[13] B. Garg, O.P. Katare, S. Beg, S. Lohan, B. Singh, Systematic development of solid selfnanoemulsifying oily formulations (S-SNEOFs) for enhancing the oral bioavailability and intestinal lymphatic uptake of lopinavir, Colloids Surf B Biointerfaces, 141 (2016) 611-622.

[14] B. Singh, S. Bandyopadhyay, R. Kapil, R. Singh, O. Katare, Self-emulsifying drug delivery systems (SEDDS): Formulation development, characterization, and applications, Crit Rev Ther Drug Carrier Syst., 26 (2009) 427-521.

[15] A.A. Date, N. Desai, R. Dixit, M. Nagarsenker, Self-nanoemulsifying drug delivery systems: Formulation insights, applications and advances, Nanomedicine (Lond). 5 (2010) 1595-1616.

[16] C.M. O'Driscoll, Lipid-based formulations for intestinal lymphatic delivery, Eur J Pharm Sci, 15 (2002) 405-415.

[17] B. Singh, R. Kumar, N. Ahuja, Optimizing drug delivery systems using systematic "design of experiments." Part I: Fundamental aspects, Crit Rev Ther Drug Carrier Syst, 22 (2005) 27-105.

[18] A.A. Sidhaye, K.C. Bhuran, S. Zambare, M. Abubaker, N. Nirmalan, K.K. Singh, Bio-inspired artemether-loaded human serum albumin nanoparticles for effective control of malaria-infected erythrocytes, Nanomedicine, 19 (2016) 19.

[19] P. Nethercote, J. Ermer, Quality by Design for analytical methods: Implications for method validation and transfer, Pharm. Tech., 36 (2012) 74-79.

[20] S. Bandyopadhyay, O.P. Katare, B. Singh, Optimized self nano-emulsifying systems of ezetimibe with enhanced bioavailability potential using long chain and medium chain triglycerides, Colloids Surf B Biointerfaces., 100 (2012) 50-61.

[21] G.R. Valicherla, K.M. Dave, A.A. Syed, M. Riyazuddin, A.P. Gupta, A. Singh, Wahajuddin, K. Mitra, D. Datta, J.R. Gayen, Formulation optimization of Docetaxel loaded self-emulsifying drug delivery system to enhance bioavailability and anti-tumor activity, Sci Rep., 6 (2016) 26895.

[22] B. Singh, L. Khurana, S. Bandyopadhyay, R. Kapil, O.P. Katare, Development of optimized selfnano-emulsifying drug delivery systems (SNEDDS) of carvedilol with enhanced bioavailability potential, Drug Deliv., 18 (2011) 599-612.

[23] B. Singh, R. Kapil, M. Nandi, N. Ahuja, Developing oral drug delivery systems using formulation by design: vital precepts, retrospect and prospects, Expert Opin Drug Deliv, 8 (2011) 1341-1360.

[24] G. Coles, B. Fuller, K. Nordquist, A. Kongslie, Using failure mode effects and criticality analysis for high-risk processes at three community hospitals, Jt Comm J Qual Patient Saf., 31 (2005) 132-140. [25] S. Beg, S.S. Jena, N. Patra Ch, M. Rizwan, S. Swain, J. Sruti, M.E. Rao, B. Singh, Development of solid self-nanoemulsifying granules (SSNEGs) of ondansetron hydrochloride with enhanced bioavailability potential, Colloids Surf B Biointerfaces, 101 (2013) 414-423.

[26] R.K. Khurana, S. Beg, D. Lal, O.P. Katare, B. Singh, Analytical quality by design approach for development of a validated bioanalytical UPLC method of docetaxel trihydrate, Curr Pharm Anal., 11 (2016) $180-192$.

[27] S. Bandyopadhyay, S. Beg, O.P. Katare, G. Sharma, B. Singh, QbD-oriented development of self-nanoemulsifying drug delivery systems (SNEDDS) of valsartan with improved biopharmaceutical performance, Curr Drug Deliv., 12 (2015) 544-563.

[28] Z. Liu, D. Liu, L. Wang, J. Zhang, N. Zhang, Docetaxel-Loaded Pluronic P123 Polymeric Micelles: in Vitro and in Vivo Evaluation, Int. J. Mol. Sci., 12 (2011) 1684-1696.

[29] A. Dahan, A. Hoffman, Rationalizing the selection of oral lipid based drug delivery systems by an in vitro dynamic lipolysis model for improved oral bioavailability of poorly water soluble drugs, $\mathrm{J}$ Control Release., 129 (2008) 1-10.

[30] H. Ali, A. Siddiqui, S. Nazzal, The effect of media composition, $\mathrm{pH}$, and formulation excipients on the in vitro lipolysis of self-emulsifying drug delivery systems (SEDDS), J Disper Sci Technol., 31 (2010) 226-232. 
[31] J. Khan, A. Hawley, T. Rades, B.J. Boyd, In situ lipolysis and synchrotron small-angle X-ray scattering for the direct determination of the precipitation and solid-state form of a poorly watersoluble drug during digestion of a lipid-based formulation, J Pharm Sci., 105 (2015) 2631-2639.

[32] A. Chaurasiya, A.K. Singh, G.K. Jain, M.H. Warsi, E. Sublet, F.J. Ahmad, G. Borchard, R.K. Khar, Dual approach utilizing self microemulsifying technique and novel P-gp inhibitor for effective delivery of taxanes, J Microencapsul., 29 (2012) 583-595.

[33] S. Martins, S. Costa-Lima, T. Carneiro, A. Cordeiro-da-Silva, E.B. Souto, D.C. Ferreira, Solid lipid nanoparticles as intracellular drug transporters: an investigation of the uptake mechanism and pathway, Int J Pharm., 430 (2012) 216-227.

[34] L.M. Negi, M. Tariq, S. Talegaonkar, Nano scale self-emulsifying oil based carrier system for improved oral bioavailability of camptothecin derivative by P-Glycoprotein modulation, Colloids Surf B Biointerfaces., 111 (2013) 346-353.

[35] X.J. Liang, C. Chen, Y. Zhao, P.C. Wang, Circumventing tumor resistance to chemotherapy by nanotechnology, Methods Mol Biol, 596 (2010) 467-488.

[36] A. Dahan, A. Hoffman, Evaluation of a chylomicron flow blocking approach to investigate the intestinal lymphatic transport of lipophilic drugs, Eur J Pharm Sci., 24 (2005) 381-388.

[37] M. Boyd, V. Risovic, P. Jull, E. Choo, K.M. Wasan, A stepwise surgical procedure to investigate the lymphatic transport of lipid-based oral drug formulations: Cannulation of the mesenteric and thoracic lymph ducts within the rat, J Pharmacol Toxicol Methods., 49 (2004) 115-120.

[38] S. Attili-Qadri, N. Karra, A. Nemirovski, O. Schwob, Y. Talmon, T. Nassar, S. Benita, Oral delivery system prolongs blood circulation of docetaxel nanocapsules via lymphatic absorption, Proc Natl Acad Sci U S A, 110 (2013) 17498-17503.

[39] M. Grove, A. Mullertz, J.L. Nielsen, G.P. Pedersen, Bioavailability of seocalcitol II: development and characterisation of self-microemulsifying drug delivery systems (SMEDDS) for oral administration containing medium and long chain triglycerides, Eur J Pharm Sci., 28 (2006) 233-242.

[40] B.T. Griffin, M. Kuentz, M. Vertzoni, E.S. Kostewicz, Y. Fei, W. Faisal, C. Stillhart, C.M. O'Driscoll, C. Reppas, J.B. Dressman, Comparison of in vitro tests at various levels of complexity for the prediction of in vivo performance of lipid-based formulations: case studies with fenofibrate, Eur J Pharm Biopharm., 86 (2014) 427-437.

[41] C.W. Pouton, Lipid formulations for oral administration of drugs: Non-emulsifying, selfemulsifying and 'self-microemulsifying' drug delivery systems, Eur J Pharm Sci, 11 (2000) S93-98.

[42] J.D. Colmenares, Development of Nanoemulsion-based Delivery Systems for Evaluation of Triglycerides Bioactivity in Caernohabditis Elegans, in: Graduate School of the, University of Massachusetts University of Massachusetts - Amherst, Amherst, 2015, pp. 2-4.

[43] T. Tran, X. Xi, T. Rades, A. Mullertz, Formulation and characterization of self-nanoemulsifying drug delivery systems containing monoacyl phosphatidylcholine, Int J Pharm., 502 (2016) 151-160.

[44] A.K. Singh, A. Chaurasiya, M. Singh, S.C. Upadhyay, R. Mukherjee, R.K. Khar, Exemestane loaded self-microemulsifying drug delivery system (SMEDDS): development and optimization, AAPS PharmSciTech, 9 (2008) 628-634.

[45] U.A. Fahmy, O.A. Ahmed, K.M. Hosny, Development and evaluation of avanafil selfnanoemulsifying drug delivery system with rapid onset of action and enhanced bioavailability, AAPS PharmSciTech., 16 (2015) 53-58.

[46] L. Sek, C.J. Porter, A.M. Kaukonen, W.N. Charman, Evaluation of the in-vitro digestion profiles of long and medium chain glycerides and the phase behaviour of their lipolytic products, J Pharm Pharmacol., 54 (2002) 29-41.

[47] O. Zupancic, A. Partenhauser, H.T. Lam, J. Rohrer, A. Bernkop-Schnurch, Development and in vitro characterisation of an oral self-emulsifying delivery system for daptomycin, Eur J Pharm Sci., 81 (2016) 129-136.

[48] D.G. Fatouros, F.S. Nielsen, D. Douroumis, L.J. Hadjileontiadis, A. Mullertz, In vitro-in vivo correlations of self-emulsifying drug delivery systems combining the dynamic lipolysis model and neuro-fuzzy networks, Eur J Pharm Biopharm., 69 (2008) 887-898.

[49] P. Bu, S. Narayanan, D. Dalrymple, X. Cheng, A.T. Serajuddin, Cytotoxicity assessment of lipidbased self-emulsifying drug delivery system with Caco-2 cell model: Cremophor EL as the surfactant, Eur J Pharm Sci., 91 (2016) 162-171.

[50] M.A. Lea, J. Pourat, R. Patel, C. desBordes, Growth inhibition of colon cancer cells by compounds affecting AMPK activity, World Gastrointest Oncol., 6 (2014) 244-252. 
[51] G. Sandri, M.C. Bonferoni, S. Rossi, F. Ferrari, S. Gibin, Y. Zambito, G. Di Colo, C. Caramella, Nanoparticles based on N-trimethylchitosan: evaluation of absorption properties using in vitro (Caco2 cells) and ex vivo (excised rat jejunum) models, Eur J Pharm Biopharm., 65 (2007) 68-77.

[52] M. Ben Reguiga, L. Bonhomme-Faivre, R. Farinotti, Bioavailability and tissular distribution of docetaxel, a P-glycoprotein substrate, are modified by interferon-alpha in rats, J Pharm Pharmacol., 59 (2007) 401-408.

[53] R. Ghaffarian, S. Muro, Models and methods to evaluate transport of drug delivery systems across cellular barriers., J Vis Exp, (2013) e50638.

[54] X. Sha, G. Yan, Y. Wu, J. Li, X. Fang, Effect of self-microemulsifying drug delivery systems containing Labrasol on tight junctions in Caco-2 cells, Eur J Pharm Sci., 24 (2005) 477-486.

[55] Z. Ujhelyi, F. Fenyvesi, J. Varadi, P. Feher, T. Kiss, S. Veszelka, M. Deli, M. Vecsernyes, I. Bacskay, Evaluation of cytotoxicity of surfactants used in self-micro emulsifying drug delivery systems and their effects on paracellular transport in Caco-2 cell monolayer, Eur J Pharm Sci., 47 (2012) 564-573.

[56] R.N. Gursoy, S. Benita, Self-emulsifying drug delivery systems (SEDDS) for improved oral delivery of lipophilic drugs, Biomed Pharmacother., 58 (2004) 173-182.

[57] N. Gursoy, J.S. Garrigue, A. Razafindratsita, G. Lambert, S. Benita, Excipient effects on in vitro cytotoxicity of a novel paclitaxel self-emulsifying drug delivery system, J Pharm Sci., 92 (2003) 2411-2418.

[58] M. Werle, Natural and synthetic polymers as inhibitors of drug efflux pumps, Pharm Res., 25 (2008) 500-511.

[59] C.Y. Kuan, T.H. Walker, P.G. Luo, C.F. Chen, Long-chain polyunsaturated fatty acids promote paclitaxel cytotoxicity via inhibition of the MDR1 gene in the human colon cancer Caco-2 cell line, J Am Coll Nutr., 30 (2011) 265-273.

[60] K. Shirakawa, K. Takara, Y. Tanigawara, N. Aoyama, M. Kasuga, F. Komada, T. Sakaeda, K. Okumura, Interaction of docetaxel ("Taxotere") with human P-glycoprotein, Jpn J Cancer Res., 90 (1999) 1380-1386.

[61] Y. Shono, H. Nishihara, Y. Matsuda, S. Furukawa, N. Okada, T. Fujita, A. Yamamoto, Modulation of intestinal P-glycoprotein function by cremophor EL and other surfactants by an in vitro diffusion chamber method using the isolated rat intestinal membranes, J Pharm Sci., 93 (2004) 877 885.

[62] A.M. Al-Mohizea, F. Zawaneh, M.A. Alam, F.I. Al-Jenoobi, G.M. El-Maghraby, Effect of pharmaceutical excipients on the permeability of P-glycoprotein substrate, J Drug Delv Sci Tech, 5 (2014) 491-495.

[63] K. Tahara, T. Sakai, H. Yamamoto, H. Takeuchi, N. Hirashima, Y. Kawashima, Improved cellular uptake of chitosan-modified PLGA nanospheres by A549 cells, Int J Pharm., 382 (2009) 198204.

[64] G.H. Chai, Y. Xu, S.Q. Chen, B. Cheng, F.Q. Hu, J. You, Y.Z. Du, H. Yuan, Transport Mechanisms of Solid Lipid Nanoparticles across Caco-2 Cell Monolayers and their Related Cytotoxicology, ACS Appl Mater Interfaces., 8 (2016) 5929-5940.

[65] L.Z. Lv, C.Q. Tong, J. Yu, M. Han, J.Q. Gao, Mechanism of enhanced oral absorption of hydrophilic drug incorporated in hydrophobic nanoparticles, Int J Nanomedicine., 8 (2013) 27092717.

[66] Q. Song, X. Wang, Q. Hu, M. Huang, L. Yao, H. Qi, Y. Qiu, X. Jiang, J. Chen, H. Chen, X. Gao, Cellular internalization pathway and transcellular transport of pegylated polyester nanoparticles in Caco-2 cells, Int J Pharm, 445 (2013) 58-68.

[67] V. Prachayasittikul, P-glycoprotein transporter in drug development, EXCLI, 15 (2016) 113-118.

[68] B. Singh, R. Singh, S. Bandyopadhyay, R. Kapil, B. Garg, Optimized nanoemulsifying systems with enhanced bioavailability of carvedilol, Colloids Surf B Biointerfaces., 101 (2013) 465-474.

[69] Y.Q. Nancy You, P.R. Ling, J.Z. Qu, B.R. Bistrian, Effects of medium-chain triglycerides, longchain triglycerides, or 2-monododecanoin on fatty acid composition in the portal vein, intestinal lymph, and systemic circulation in rats, J Parenter Enteral Nutr, 32 (2008) 169-175.

[70] H. Kim, Y. Kim, J. Lee, Liposomal formulations for enhanced lymphatic drug delivery, Asian J Pharm Sci, 8 (2013) 96-103.

[71] A.S. Chudasama, V.V. Patel, M. Nivsarkar, K.K. Vasu, C.J. Shishoo, In vivo evaluation of self emulsifying drug delivery system for oral delivery of nevirapine, Indian J Pharm Sci., 76 (2014) 218224. 
[72] A. Ali Khan, J. Mudassir, N. Mohtar, Y. Darwis, Advanced drug delivery to the lymphatic system: lipid-based nanoformulations, Int J Nanomed, 8 (2013) 2733-2744.

[73] C.J. Porter, W.N. Charman, Intestinal lymphatic drug transport: an update, Adv Drug Deliv Rev., 50 (2001) 61-80.

[74] W. Wu, Y. Wang, L. Que, Enhanced bioavailability of silymarin by self-microemulsifying drug delivery system, Eur J Pharm Biopharm., 63 (2006) 288-294.

[75] E.S. Swenson, W.J. Curatolo, Intestinal permeability enhancement for proteins, peptides and other polar drugs: mechanisms and potential toxicity., Adv Drug Deliv Rev., 8 (1992) 39-92.

[76] J. Tang, J. Sun, Z. He, Self-emulsifying drug delivery systems: Strategy for improving oral delivery of poorly soluble drugs, Curr Drug Ther, 2 (2007) 85-93.

[77] T. Bansal, G. Mishra, M. Jaggi, R.K. Khar, S. Talegaonkar, Effect of P-glycoprotein inhibitor, verapamil, on oral bioavailability and pharmacokinetics of irinotecan in rats, Eur J Pharm Sci., 36 (2009) 580-590.

[78] L. Li, T. Yi, C.W. Lam, Interactions between human multidrug resistance related protein (MRP2; $\mathrm{ABCC} 2$ ) and excipients commonly used in self-emulsifying drug delivery systems (SEDDS), Int $\mathbf{J}$ Pharm, 447 (2013) 192-198.

[79] M. Chitneni, K.K. Peh, D. Darwis, M. Abdulkarim, G.Z. Abdullah, M.J. Qureshi, Intestinal permeability studies of sulpiride incorporated into self-microemulsifying drug delivery system (SMEDDS), Pak J Pharm Sci., 24 (2011) 113-121.

[80] Q. Xing, J. Song, X. You, D. Xu, K. Wang, Q. Guo, P. Li, C. Wu, H. Hu, Microemulsions containing long-chain oil ethyl oleate improve the oral bioavailability of piroxicam by increasing drug solubility and lymphatic transportation simultaneously, Int J Pharm., 511 (2016) 709-718.

[81] S.M. Caliph, W.N. Charman, C.J. Porter, Effect of short-, medium-, and long-chain fatty acidbased vehicles on the absolute oral bioavailability and intestinal lymphatic transport of halofantrine and assessment of mass balance in lymph-cannulated and non-cannulated rats, J Pharm Sci., 89 (2000) 1073-1084. 


\section{Figure captions:}

Figure 1: Ternary phase diagrams: (A-D) Long chain glycerides: LCG (with Smix ratios of (A)1:0; (B) 1:1; (C) $2: 1$; (D) $3: 1$ and (E-H) Medium chain glycerides: MCG (with Smix ratios of (A) 1:0; (B) 1:1; (C) 2:1; (D) $3: 1$.

Figure 2(i) Half-normal plots and Pareto charts extracted resulting after application of Placket-Burman design for long chain glycerides self-nanoemulsifyinglipidic systems (LCG-SNELS) depicting the influence of material attributes (MAs) and process parameters (PPs) on the critical quality attributes (CQAs), (A) globule size: $\mathrm{D}_{\mathrm{nm}}$, (B) percent dissolutionefficiency in 5 min: $\% \mathrm{DE}_{5 \min }$, (C) emulsification time: $\mathrm{T}_{\mathrm{emul}}$, (D) Drug release in 15 min $\operatorname{Rel}_{15 \min }$ (E) Mean dissolution time: MDT.

Figure 2(ii) Half-normal plots and Pareto charts resulting after application of Fractional factorial design medium chain glycerides self-nanoemulsifyinglipidic systems (MCG-SNELS) depicting the influence of material attributes (MAs) and process parameters (PPs) on the critical quality attributes (CQAs), (A) globule size: $\mathrm{D}_{\mathrm{nm}},(\mathrm{B})$ percent dissolution efficiency in $5 \mathrm{~min}$ : $\% \mathrm{DE}_{5 \mathrm{~min}},(\mathrm{C})$ emulsification time: $\mathrm{T}_{\mathrm{emul}}$, (D) drug release in $15 \mathrm{~min}: \operatorname{Rel}_{15 \min }(\mathrm{E})$ mean dissolution time: MDT.

Figure 3: 3D-Response surface plots showing the influence of (A) Maisine-35-1, (B) Tween 80, (C) Transcutol $\mathrm{HP}$ on the CQAs viz.(i) globule size $\left(\mathrm{D}_{\mathrm{nm}}\right)$, (iii) emulsification time $\left(\mathrm{T}_{\mathrm{emul}}\right)$, (v) percent drug release in $15 \mathrm{~min}\left(\operatorname{Rel}_{15 \mathrm{~min}}\right)$, (vii) permeability in $45 \mathrm{~min}\left(\mathrm{Perm}_{45 \mathrm{~min}}\right)$; Likewise, the other 3-D response surface plots show the influence of (A) Capmul MCM, (B) Tween 80, (C) Transcutol HP on the CQAs viz. (ii) globule size $\left(\mathrm{D}_{\mathrm{nm}}\right)$, (iv) emulsification time $\left(\mathrm{T}_{\text {emul }}\right)$, (vi) percent drug release in $15 \mathrm{~min}\left(\mathrm{Rel}_{15 \mathrm{~min}}\right)$, (viii) permeability in 45 mins $\left(\right.$ Perm $\left._{45 \mathrm{~min}}\right)$.

Figure 4: Cumulative in vitro drug release profile of DTH from (A) LCG SNELS and (B) MCG SNELS formulations prepared as per the I- and D-Optimal Mixture design, respectively; Values expressed in mean $\pm \mathrm{SD}(\mathrm{n}=3)$.

Design space overlay plot for optimized (C) LCG-SNELS and (D) MCG-SNELS across the twodimensional experimental domain; TEM image of (E) LCG-SNELS and (F) MCG-SNELS.

Figure 5 (A) Comparative consumption of $\mathrm{NaOH}$ as a function of time for LCG and MCG SNELS (mean \pm SD, $\mathrm{n}=3$ ) (B) The \% of DTH release from both the SNELS formulation into the aqueous phase as a function of time (mean $\pm \mathrm{SD}, \mathrm{n}=3$ ) (C) Appearance of both the formulations after lipolysis (i) MCG SNELS (ii) LCG SNELS.

Figure 6: Cell viability assay on Caco-2 cells following treatment with DTH, LCG- SNELS and MCG-SNELS at (A) $24 \mathrm{~h}$, (B) $48 \mathrm{~h}$, (C) $72 \mathrm{~h}$. (D) Bidirectional permeation study of various formulations of DTH on Caco-2 cells.

Figure 7: Fluorsecent images of time dependent cellular uptake at (A) Control cells, (B) MCG-SNELS at $1 \mathrm{~h}$, (C) LCG-SNELS at $1 \mathrm{~h}$, (D) MCG-SNELS at $4 \mathrm{~h}$, (E) LCG-SNELS at $4 \mathrm{~h}$; (F)Fluorescence intensity at various time points for Rh-123 loaded LCG- and MCG-SNELS in Caco-2 cells, a quantitative measurement.

Figure 8: Influence of different endocytosis inhibitors on cellular uptake of Rh-123 loaded LCG- and MCGSNELS at $1 \mathrm{~h}$ in Caco-2 cells, a qualitative measurement; (A) Control cells (B) without presence of inhibitor (C) at $4{ }^{\circ} \mathrm{C}$; after treatment with (D) Sucrose (E) Nystatin (F) Cytochalasin B; (G) Fluorescence intensity at $1 \mathrm{~h}$ for Rh-123 loaded LCG- and MCG-SNELS in Caco-2 cells after treatment with various endocytotic inhibitors, a quantitative measurement.

Figure 9: (A) Effect of LCG- and MCG-SNELS on efflux of MDRI transporter Rh-123 and BCRP transporterDiOC 2 as measured by fluorescence in Caco-2 cells (B) Lymph drug concentration $(\mu \mathrm{g} / \mathrm{mL})$ of LCG- and MCG-SNELS vis-a-vis plain DTH; (C) Percent increase in the permeability paramaeters (i.e., $\mathrm{P}_{\text {eff }}$ and $\mathrm{P}_{\text {wall }}$ ), and absorption parameters (i.e., Fa and $\mathrm{A}_{\mathrm{n}}$ ) of LCG-SNELS and MCG SNELS visà-vis pure DTH and DTH with verapamil; (D) Histopathology of small intestine: (i) Control $(\times 200)$; (ii) Plain DTH (× 200); (iii) LCG-SNELS (× 200); (iv) MCG-SNELS $(\times 200)$; Small intestine showing normal tall villi and intact brush borders. No other significant pathological changes were observed; (E) Plasma concentration time profile versus time for optimized LCG- and MCG-SNELS vis-à-vis pure DTH.

Figure 10: Diagram portraying the intestinal uptake of LCG- and MCG-SNELS 


\section{Table captions:}

Table 1: Pharmacokinetic parameters of DTH obtained from plasma level studies in rats after oral administration of various treatment formulations

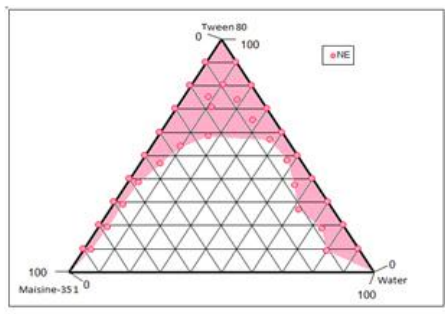

(A)

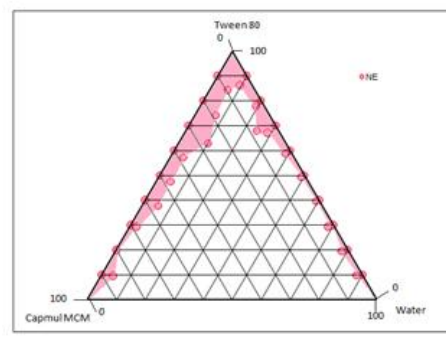

(E)

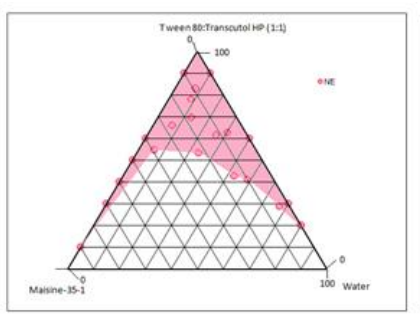

(B)

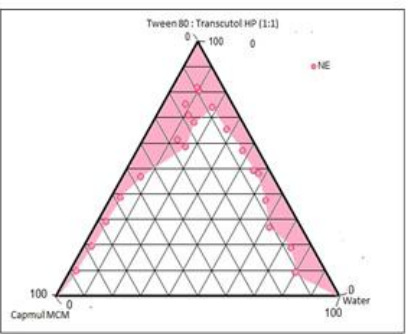

(F)

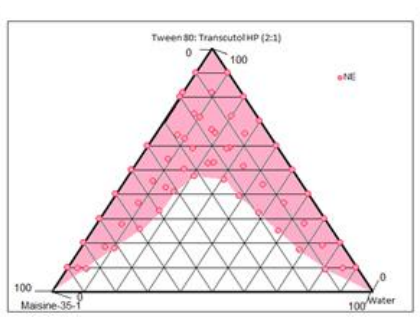

(c)

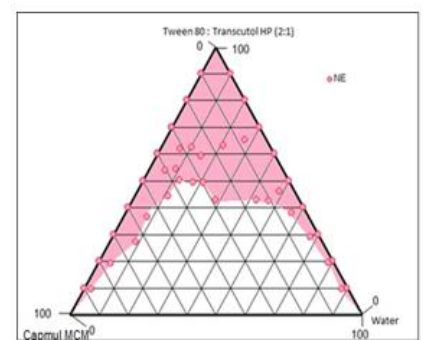

(G)

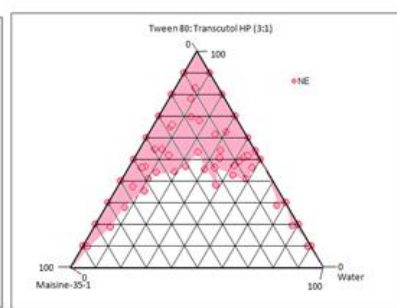

(D)

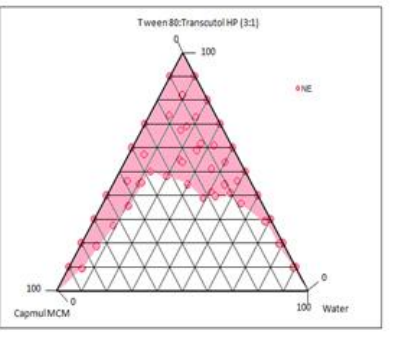

(H)

Figure 1 


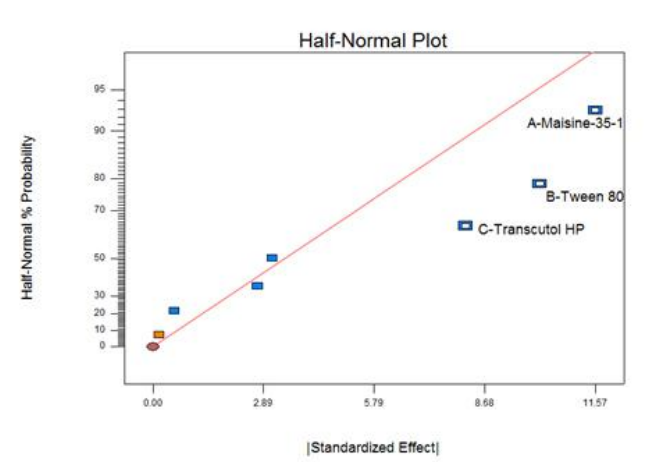

(A)
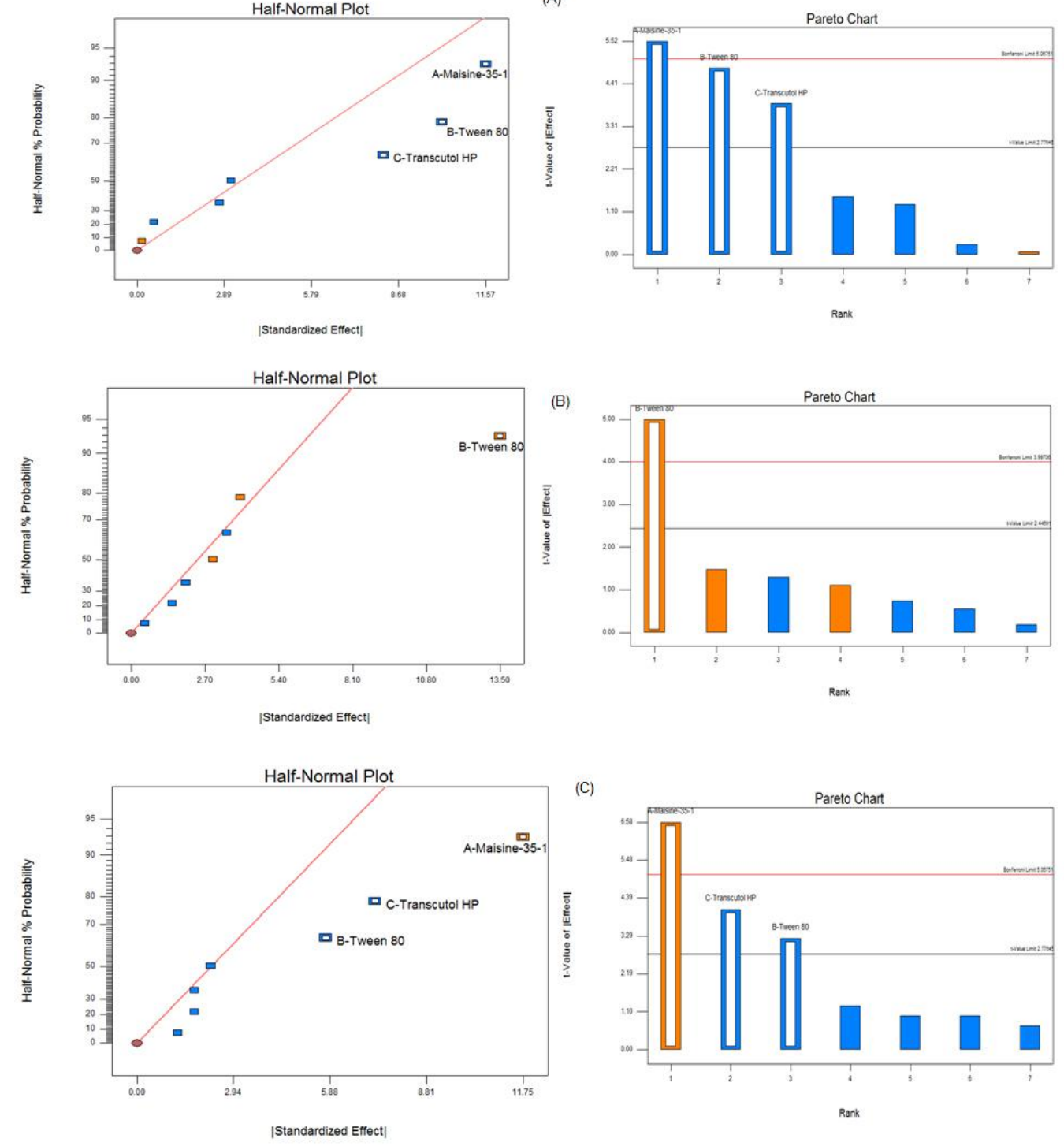

(C)
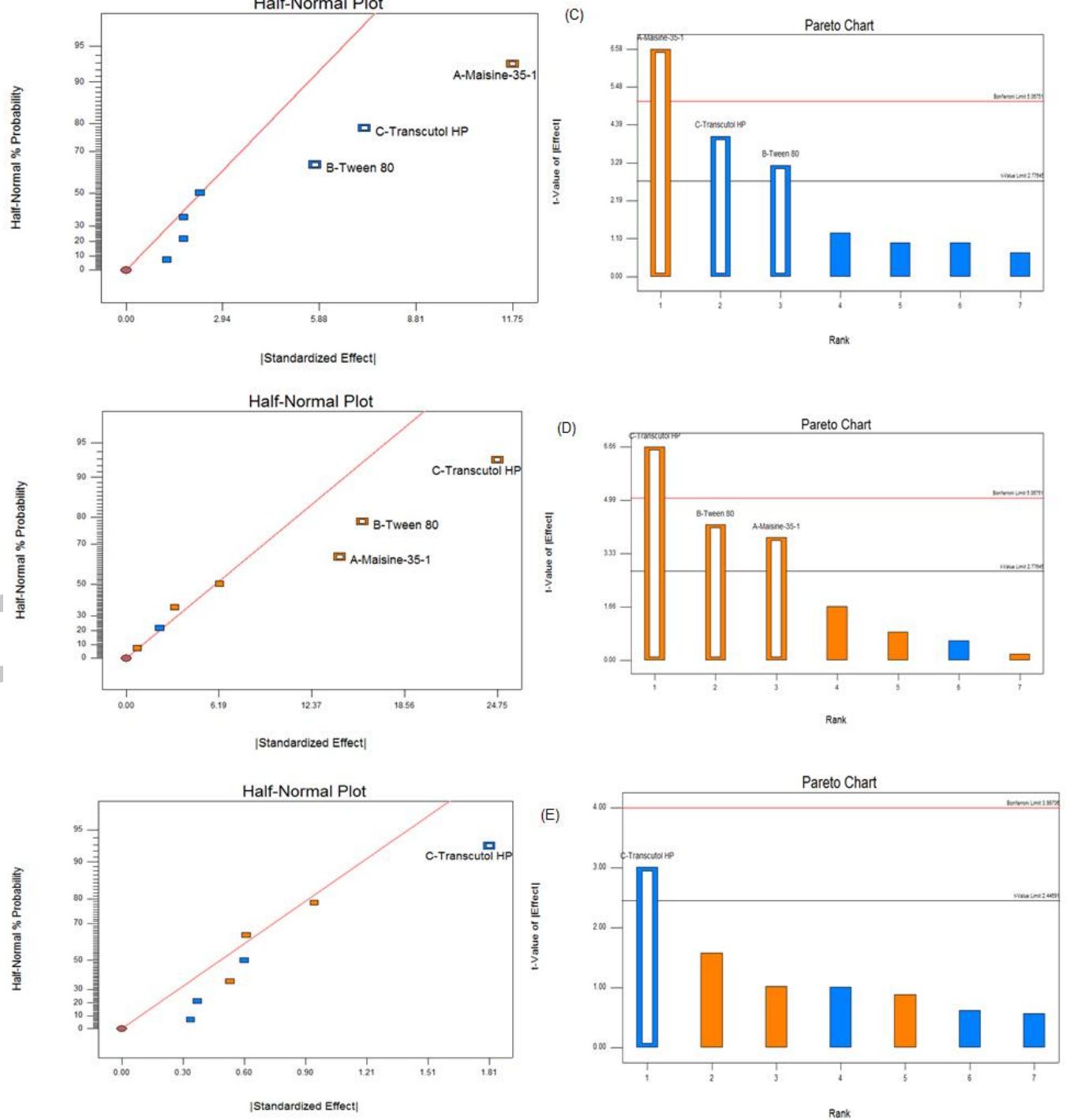

Figure 2(i) 

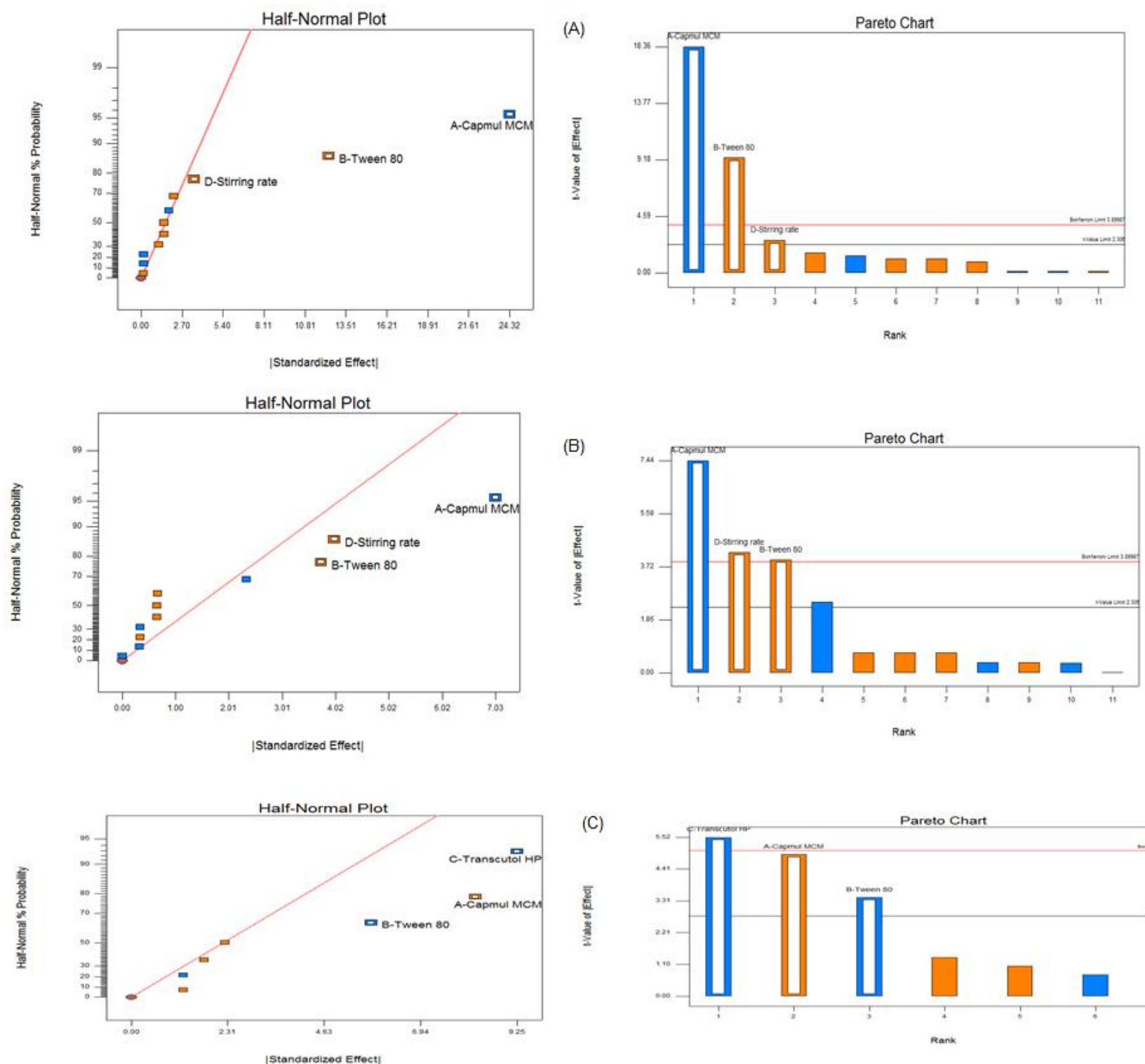

(C)
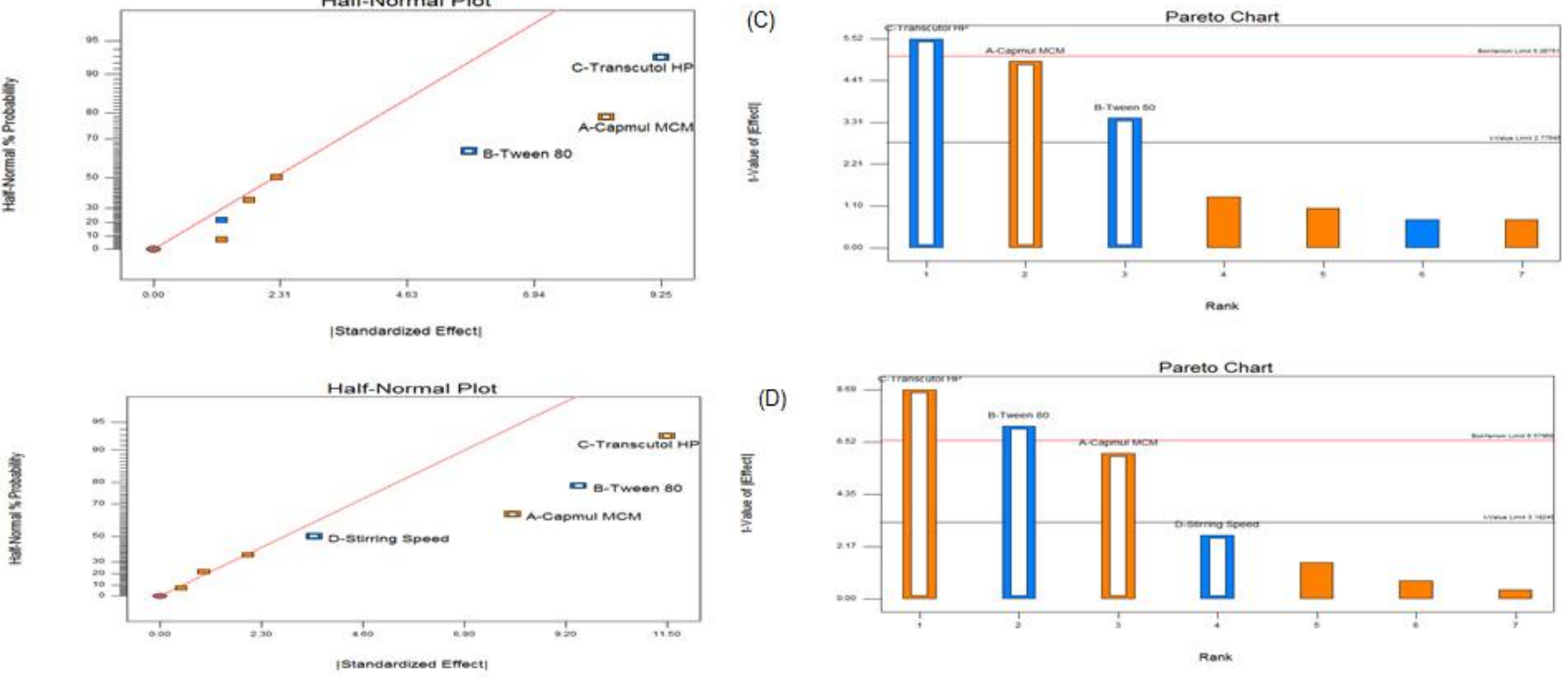

(D)
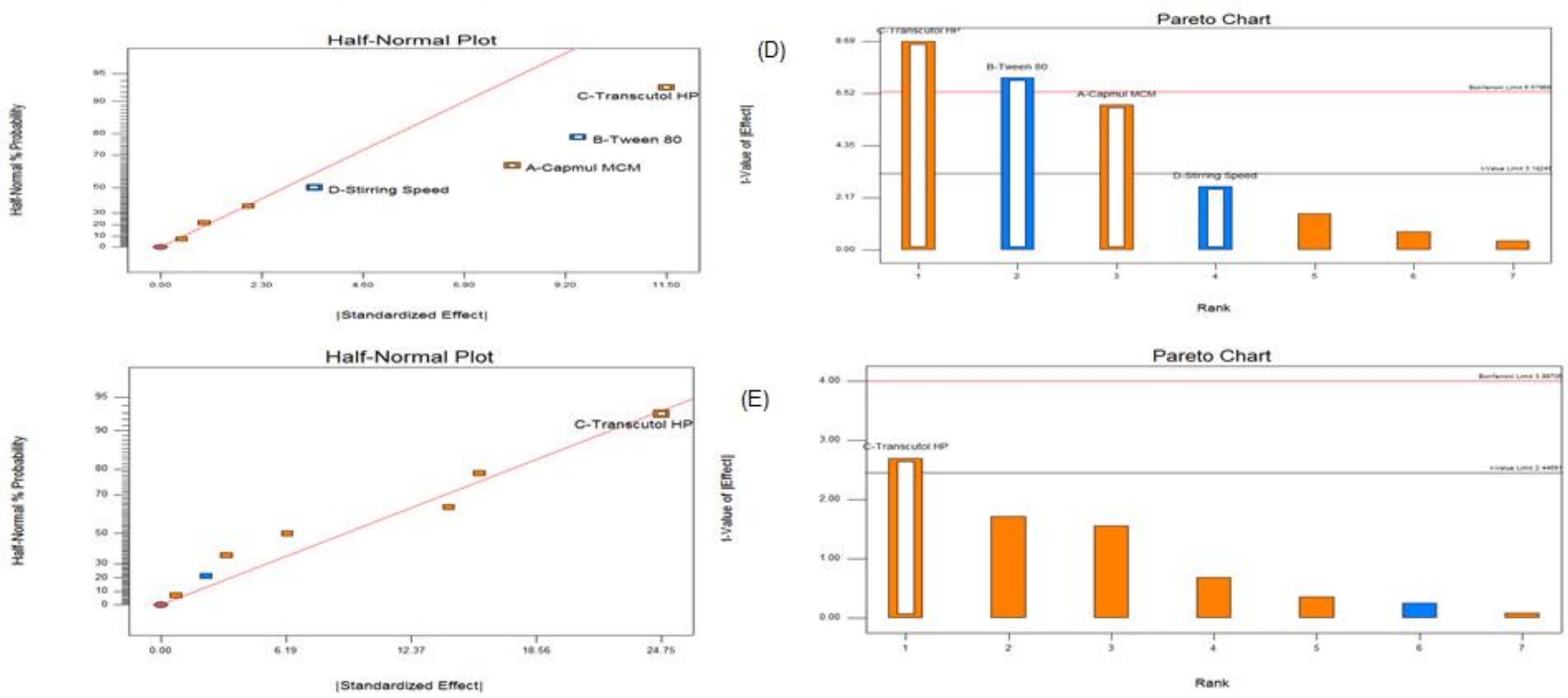

Figure 2(ii) 

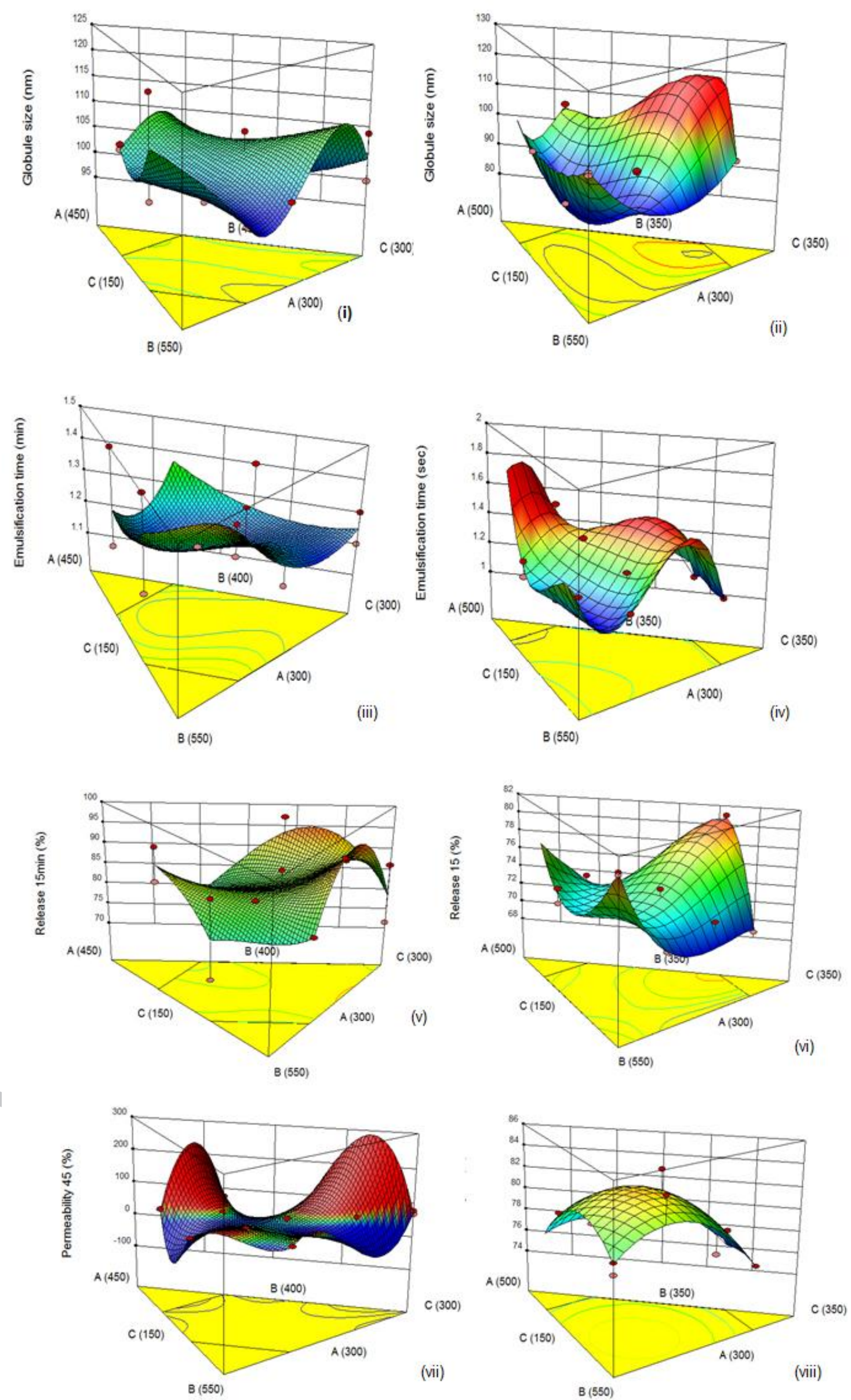

Figure 3 


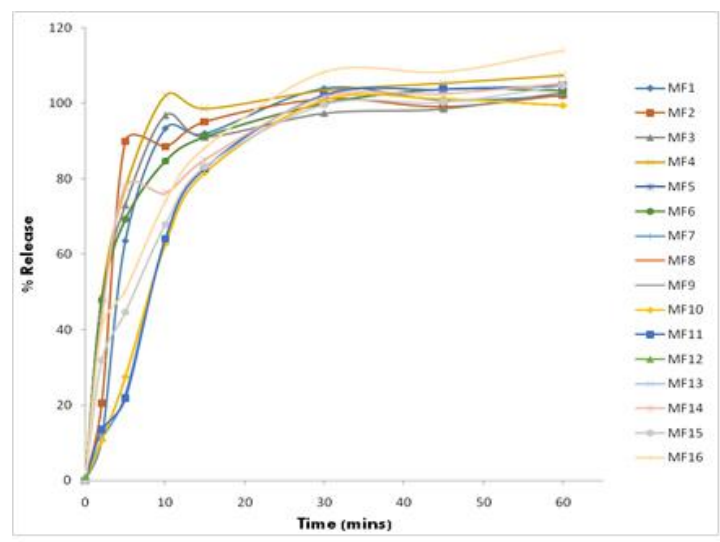

(A)

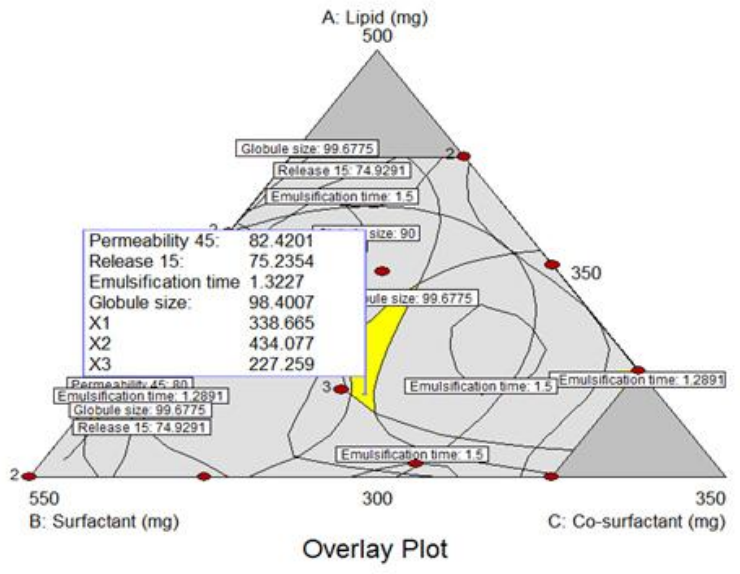

(C)

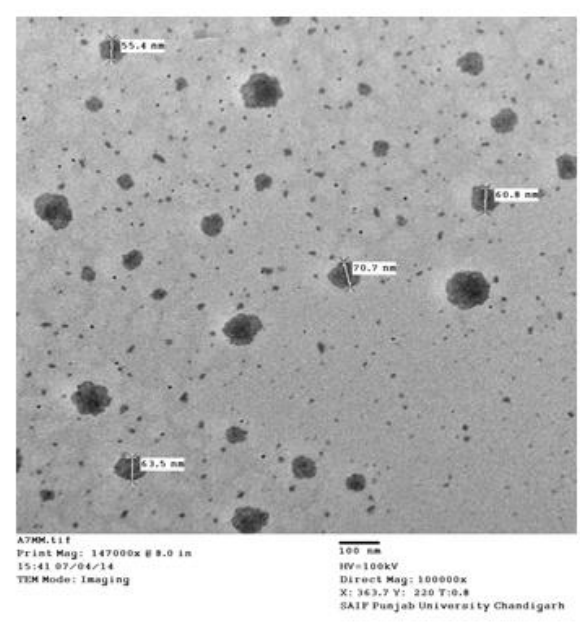

(E)

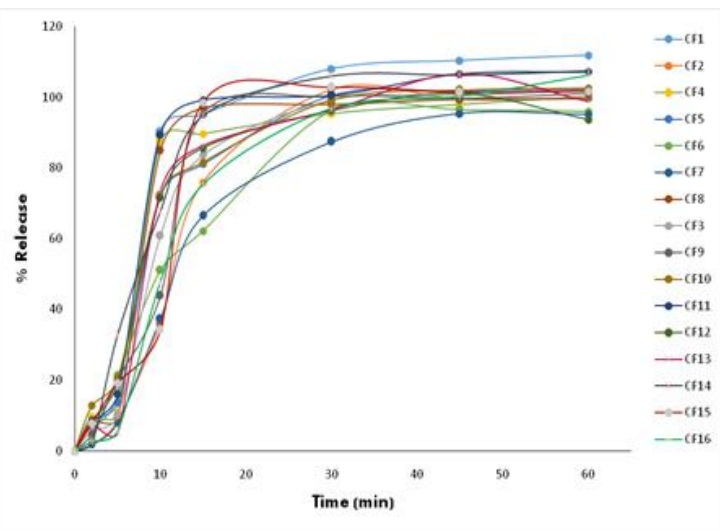

(B)

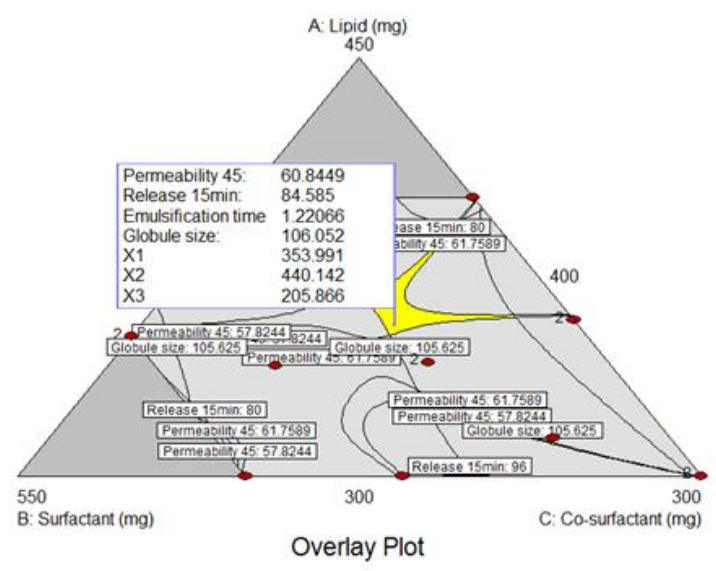

(D)

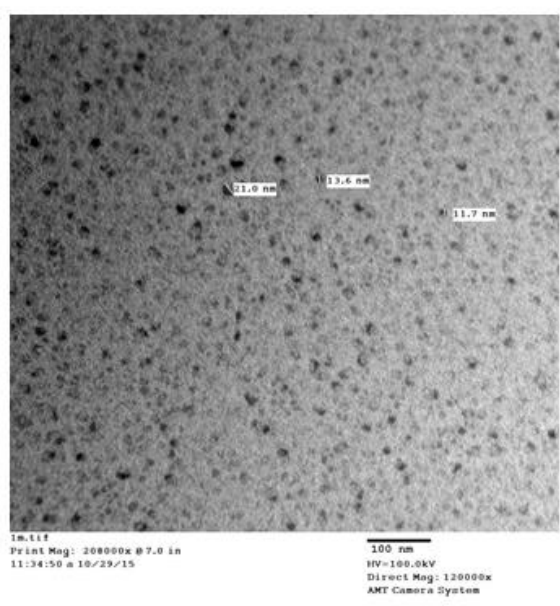

(F)

Figure 4 


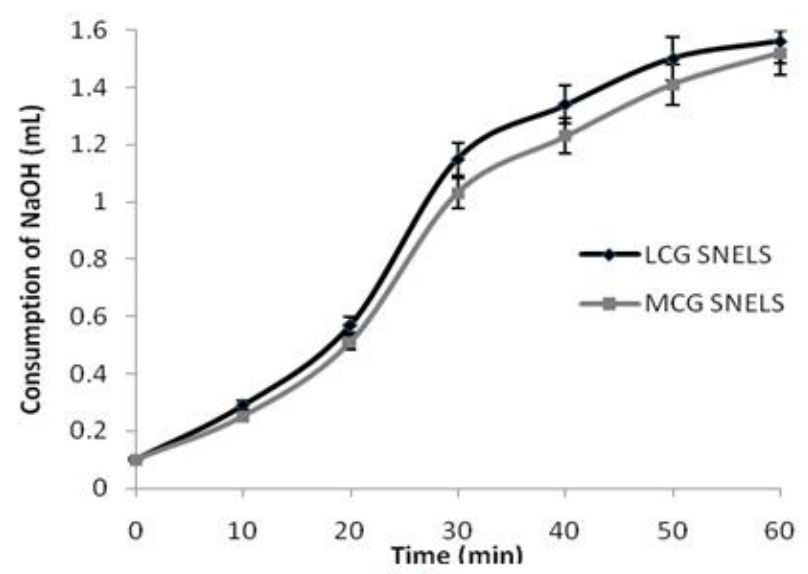

(A)

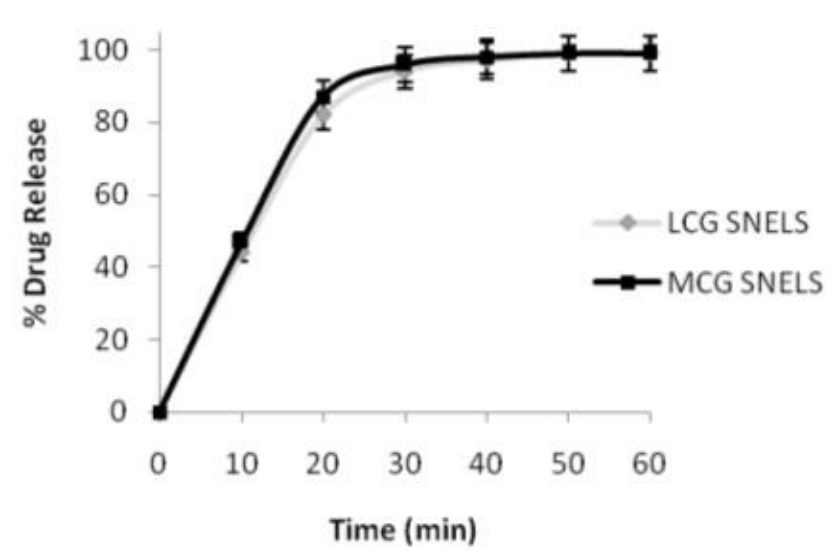

(B)

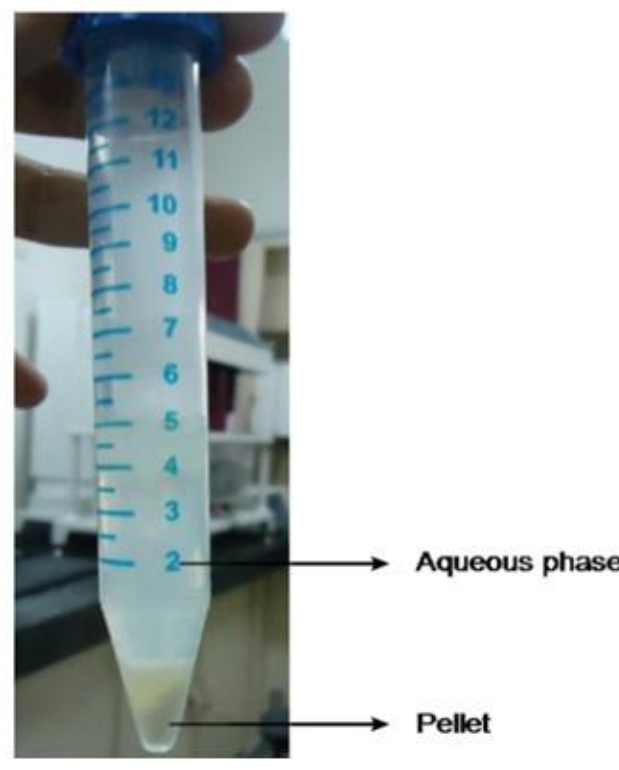

(i)

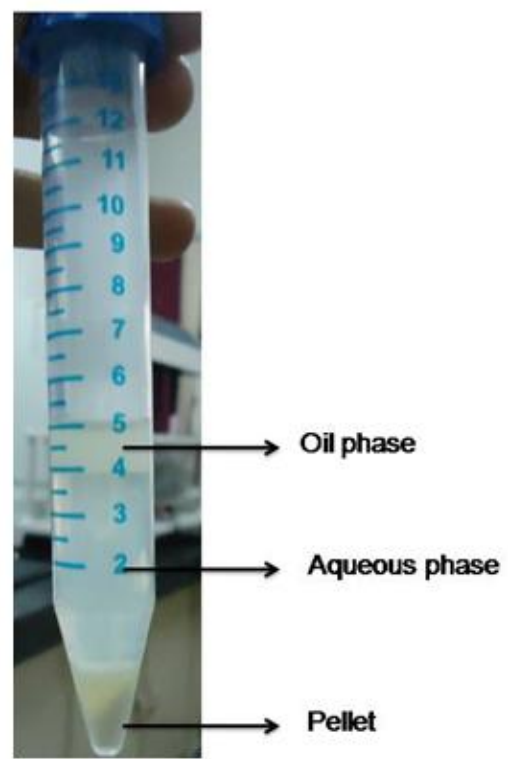

(ii)

(C)

Figure 5 


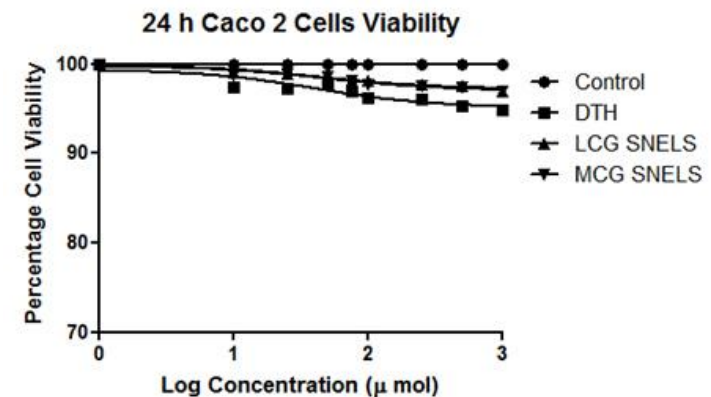

(A)

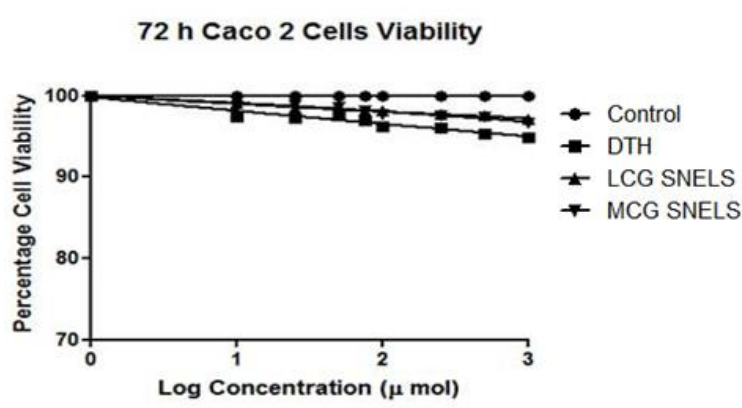

(C)

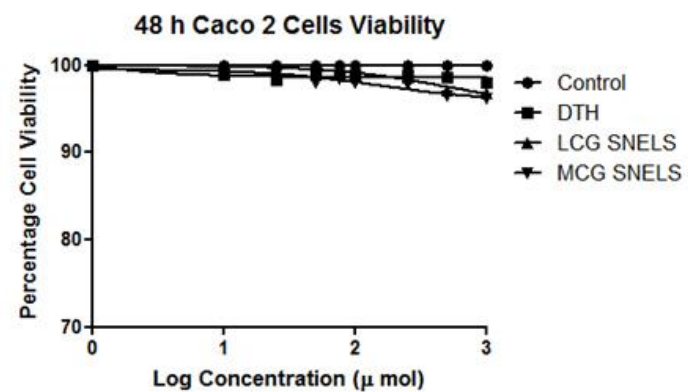

(B)

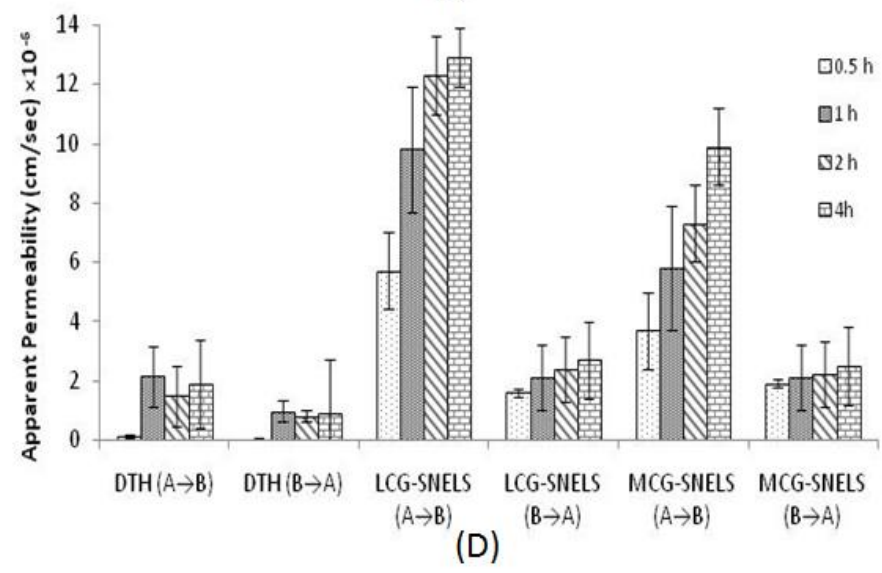

Figure 6 

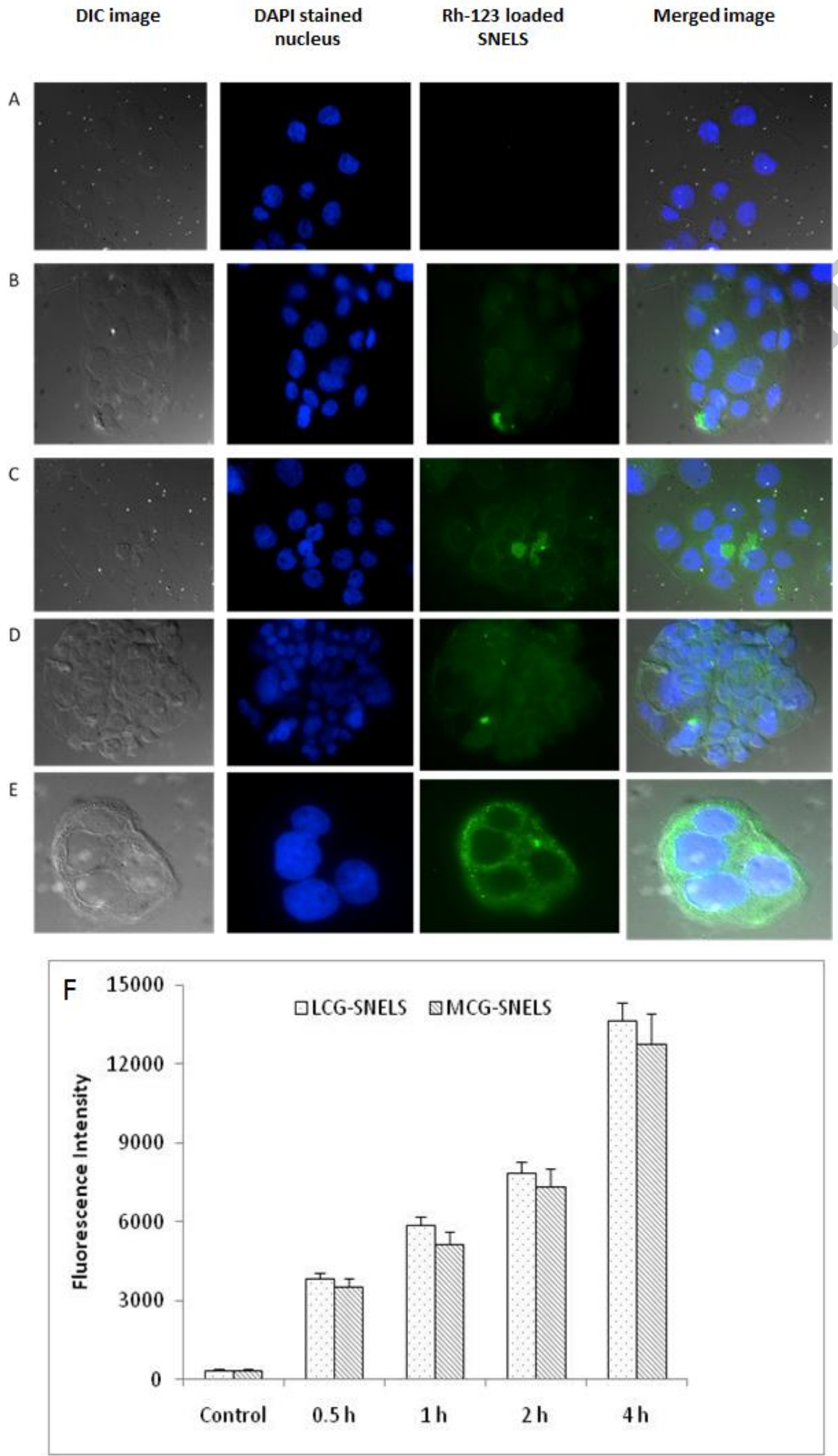

Figure 7 


\section{ACCEPTED MANUSCRIPT}

LCG-SNELS
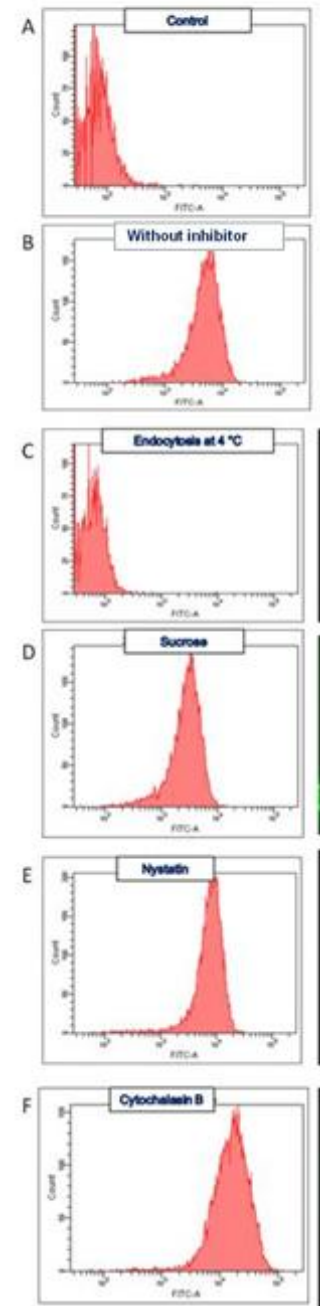
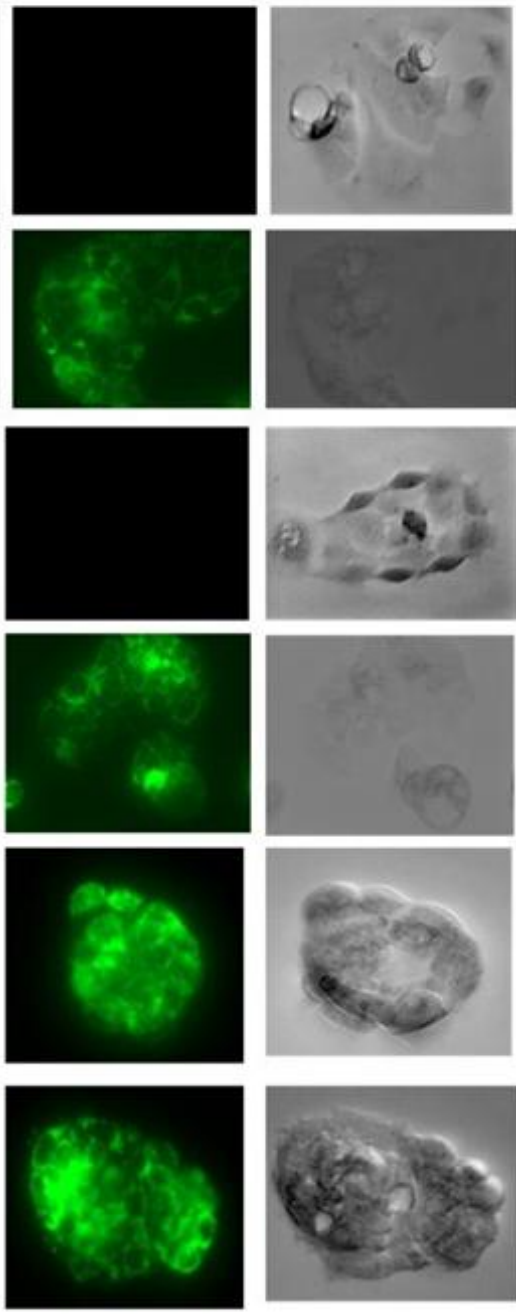

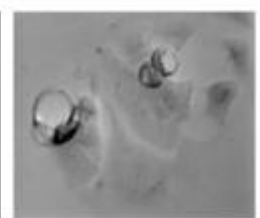

MCG-SNELS
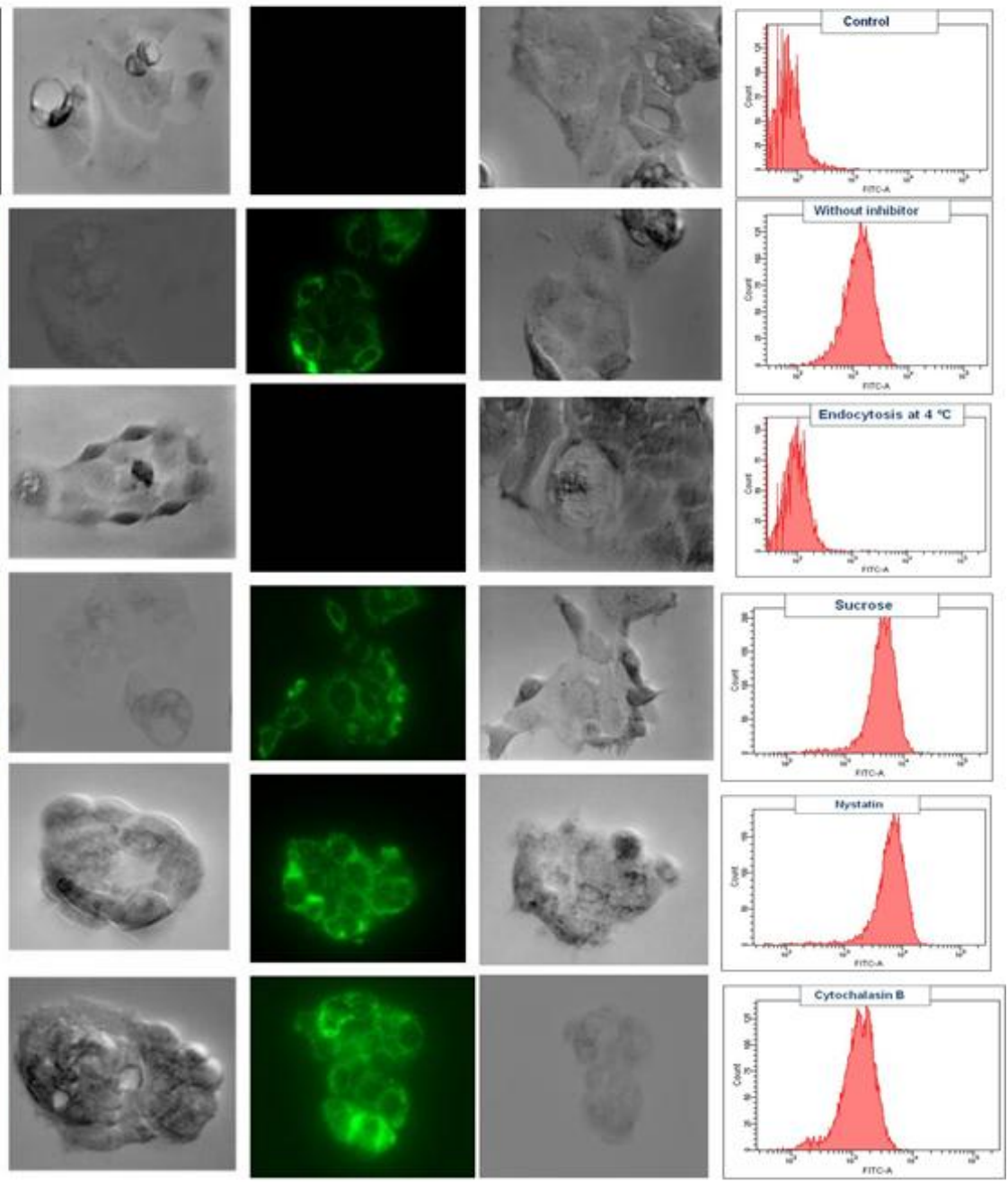

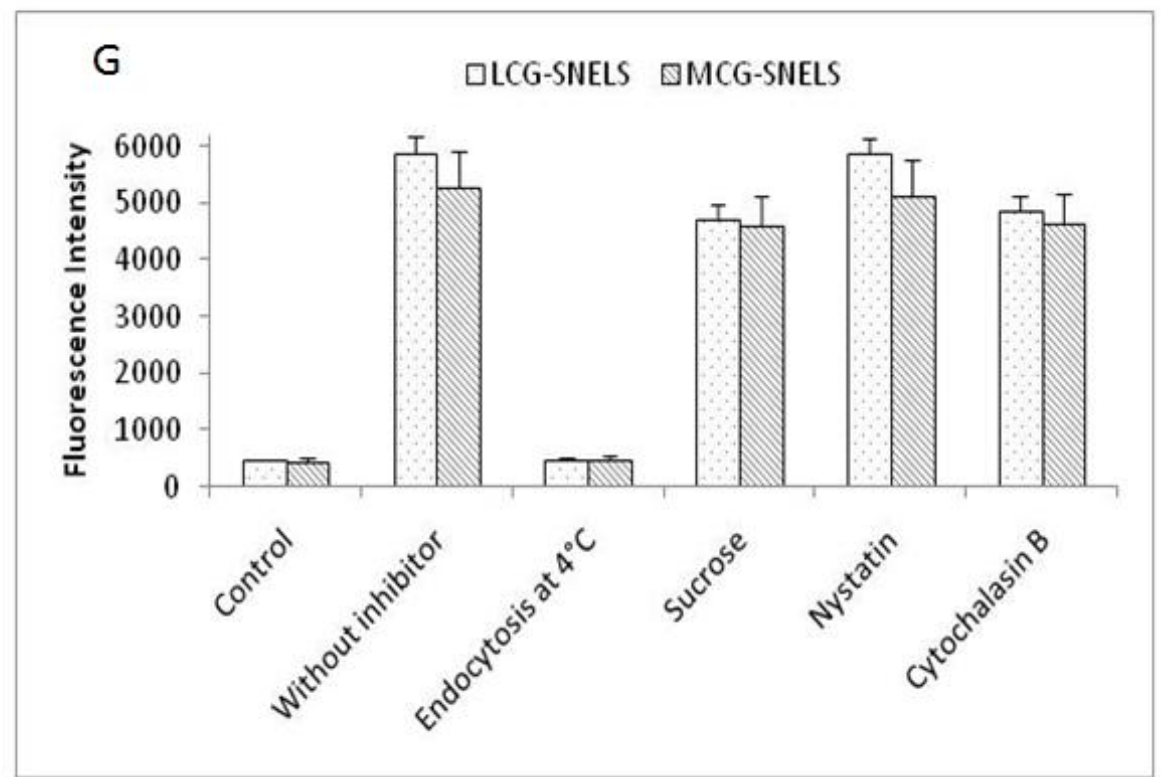

Figure 8 

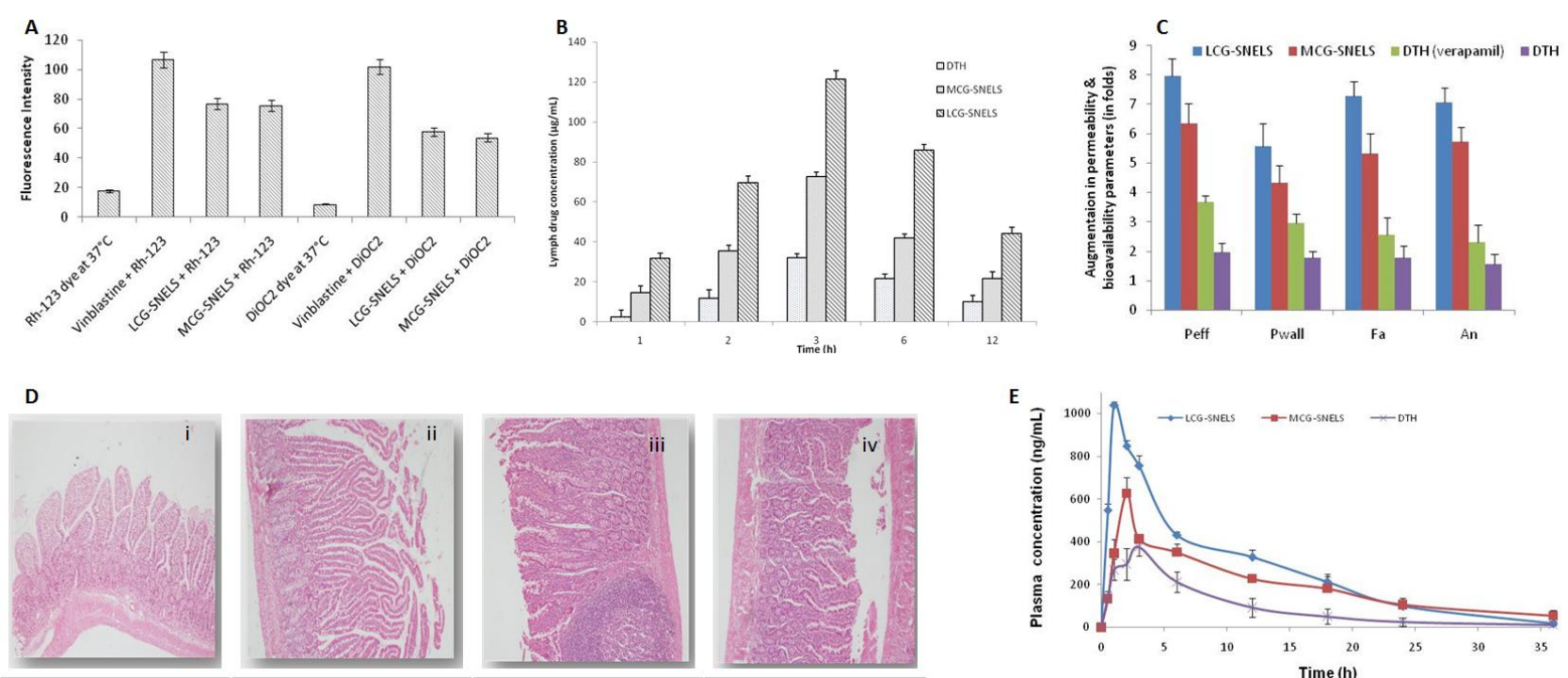

Figure 9 


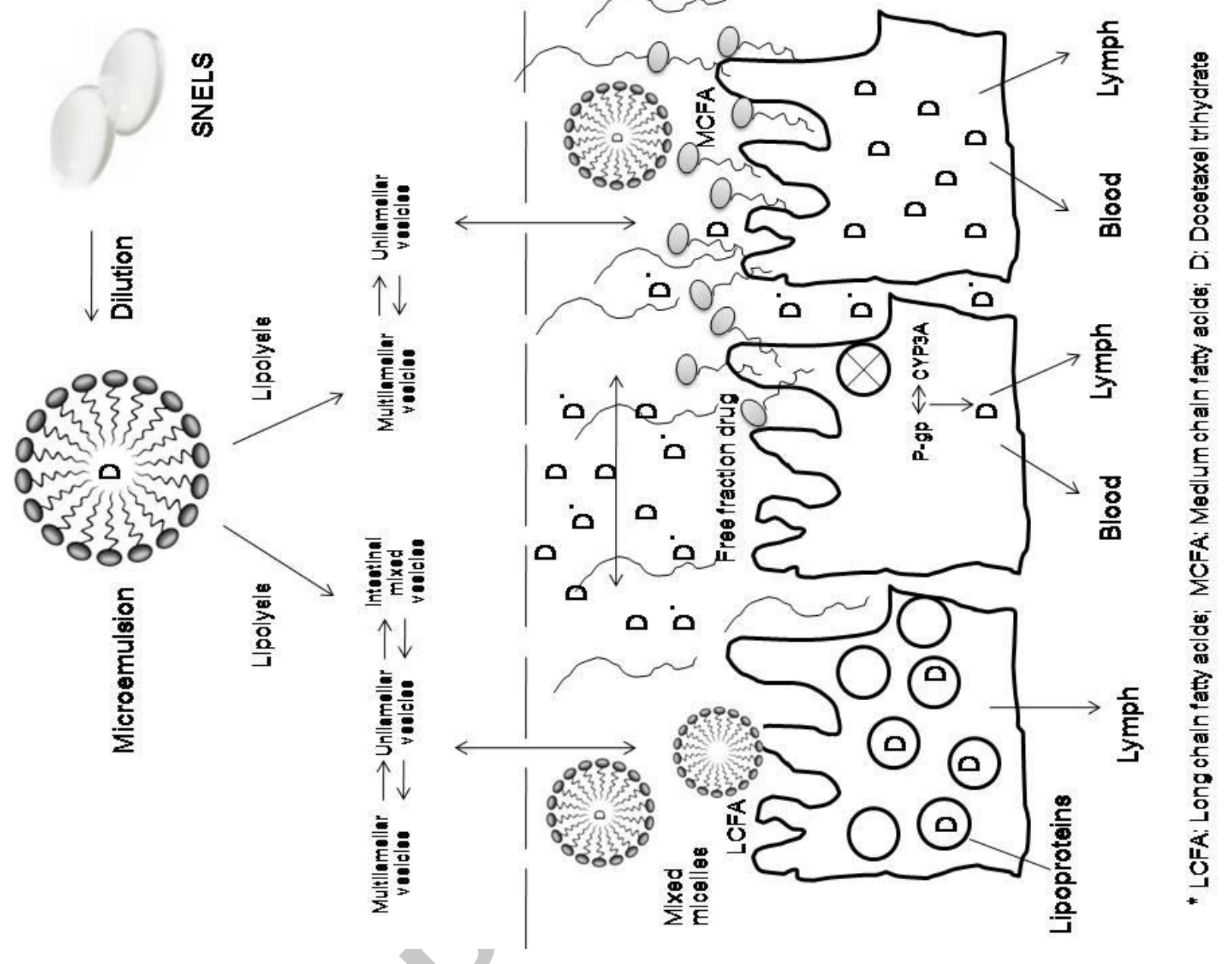

Figure 10 
Table 1: Various compartmental modelling parameters

\begin{tabular}{|c|c|c|c|c|c|}
\hline \multirow[b]{2}{*}{$\begin{array}{c}\text { Treatment } \\
\text { Formulations }\end{array}$} & \multicolumn{5}{|c|}{ Pharmacokinetics metrics } \\
\hline & $\begin{array}{l}\mathrm{C}_{\max } \\
\left(\mathrm{ng} \mathrm{mL}^{-1}\right)\end{array}$ & $\begin{array}{l}\mathbf{A U C} C_{\text {last }} \\
\left.\text { (ng.h/mL } / \mathbf{m L}^{-1}\right)\end{array}$ & $\mathbf{K a}\left(\mathbf{h}^{-1}\right)$ & $\mathbf{T}_{\max }(\mathbf{h})$ & MRT (h) \\
\hline LCG-SNELS & $1597.2 \pm 59.4$ & $9197.7 \pm 46.9$ & $0.213 \pm 0.023$ & $1.42 \pm 0.28$ & $18.47 \pm 2.56$ \\
\hline MCG-SNELS & $612.5 \pm 78.4$ & $7425.8 \pm 66.9$ & $0.141 \pm 0.021$ & $2.74 \pm 0.42$ & $16.56 \pm 1.45$ \\
\hline DTH & $346.9 \pm 58.4$ & $847.2 \pm 106.0$ & $0.112 \pm 0.013$ & $3.27 \pm 0.30$ & $11.56 \pm 1.45$ \\
\hline
\end{tabular}

Data represented as Mean \pm SD $(n=3)$ 